Linköping Studies in Science and Technology

Licentiate Thesis No. 1847

Ebrahim Chalangar

\title{
Graphene-based \\ nanocomposites for \\ electronics and photocatalysis
}

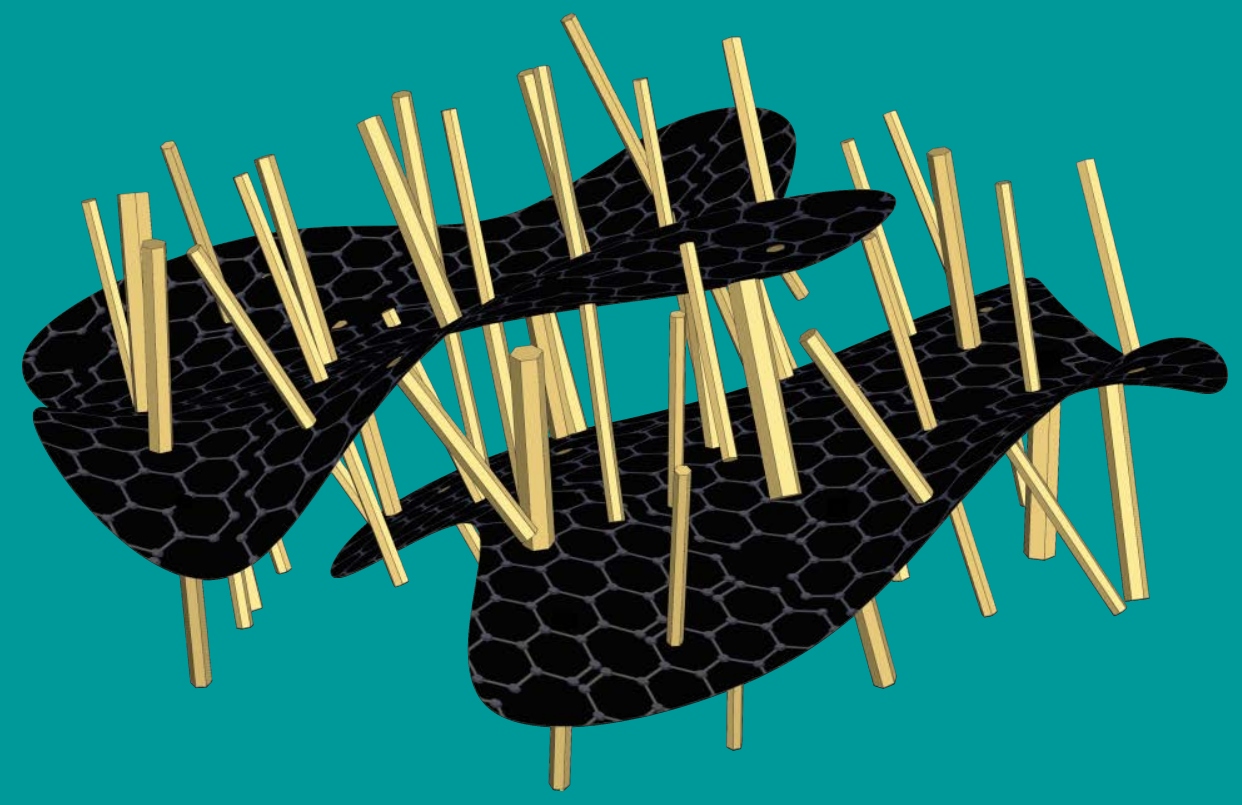


Linköping Studies in Science and Technology, Licentiate Thesis

No. 1847

\title{
Graphene-based nanocomposites for electronics and photocatalysis
}

\author{
Ebrahim Chalangar
}

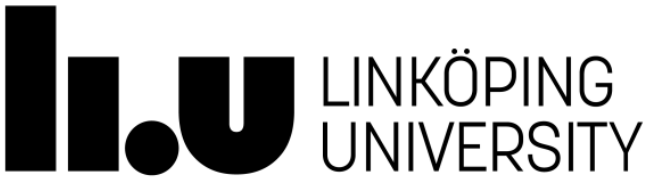

LICENTIATE THESIS

To be defended on June 13, 2019 at 14:15 in K3

Department of Science and Technology

Division of Physics and Electronics

Linköpings University, Sweden

Norrköping 2019 
(C) Ebrahim Chalangar, 2019

Printed in Sweden by LiU-Tryck, Linköping, Sweden, 2019

ISSN 0280-7971

ISBN 978-91-7685-040-4 


\section{Abstract}

The development of future electronics depends on the availability of suitable functional materials. Printed electronics, for example, relies on access to highly conductive, inexpensive and printable materials, while strong light absorption and low carrier recombination rates are demanded in photocatalysis industry. Despite all efforts to develop new materials, it still remains a challenge to have all the desirable aspects in a single material. One possible route towards novel functional materials, with improved and unprecedented physical properties, is to form composites of different selected materials.

In this work, we report on hydrothermal growth and characterization of graphene/zinc oxide ( $\mathrm{GR} / \mathrm{ZnO}$ ) nanocomposites, suited for electronics and photocatalysis application. For conductive purposes, highly $\mathrm{Al}$-doped $\mathrm{ZnO}$ nanorods grown on graphene nanoplates (GNPs) prevent the GNPs from agglomerating and promote conductive paths between the GNPs. The effect of the ZnO nanorod morphology and GR dispersity on the nanocomposite conductivity and GR/ZnO nanorod bonding strength were investigated by conductivity measurements and optical spectroscopy. The inspected samples show that growth in high $\mathrm{pH}$ solutions promotes a better graphene dispersity, higher doping and enhanced bonding between the GNPs and the $\mathrm{ZnO}$ nanorods. Growth in low $\mathrm{pH}$ solutions yield samples characterized by a higher conductivity and a reduced number of surface defects.

In addition, different $\mathrm{GR} / \mathrm{ZnO}$ nanocomposites, decorated with plasmonic silver iodide (AgI) nanoparticles, were synthesized and analyzed for solar-driven photocatalysis. The addition of Ag/AgI generates a strong surface plasmon resonance effect involving metallic $\mathrm{Ag}^{\mathrm{O}}$, which redshifts the optical absorption maximum into the visible light region enhancing the photocatalytic performance under solar irradiation. A wide range of characterization techniques including, electron microscopy, photoelectron spectroscopy and x-ray diffraction confirm a successful formation of photocatalysts.

Our findings show that the novel proposed GR-based nanocomposites can lead to further development of efficient photocatalyst materials with applications in removal of organic pollutants, or for fabrication of large volumes of inexpensive porous conjugated GR-semiconductor composites.

Keywords: graphene, zinc oxide, silver iodine, plasmonics, nanocomposites, conjugated electronics, photocatalysis, photodegradation

Department of Science and Technology

Linköpings University, Sweden 


\section{Acknowledgements}

This work was done in collaboration with Halmstad University. I would like to thank all my colleagues and my friends at Halmstad University and Linköping University. Special thanks to my supervisor Håkan Pettersson that I learned many things from him. Also, I appreciate my cosupervisors Magnus Willander and Omer Nur for their great helps.

And tremendous thanks to my wife for her love and her support.

Ebrahim Chalangar,

Norrköping, May 2019. 


\section{List of Papers}

I. E. Chalangar, H. Machhadani, S. Lim, K. F. Karlsson, O. Nur, M. Willander, H. Pettersson, "Influence of morphology on electrical and optical properties of graphene/Al-doped $\mathrm{ZnO}-$ nanorod composites," Nanotechnology, vol. 29, no. 41, p. 415201, 2018.

II. R. E. Adam $\neq$, E. Chalangar $\neq$, M. Pirhashemi, G. Pozina, X. Liu, J. Palisaitis, P. O. Persson, H. Pettersson, M. Willander and O. Nur, "Graphene-based plasmonic nanocomposites for highly enhanced solar-driven photocatalytic activities", ACS Omega, Submitted. ( $¥$ Both co-first authors) 


\section{Abbreviations}

\begin{tabular}{ll} 
1LG & Single layer graphene \\
2LG & Bilayer graphene \\
AgI & Silver iodide \\
AZO & Al-doped ZnO \\
CL & Cathodoluminescence \\
CNT & Carbo nanotubes \\
CR & Congo red \\
CVD & Chemical vapor deposition \\
EDS & Energy dispersive x-ray spectroscopy \\
FLG & Few-layered graphene \\
GNPs & Graphene nanoplates \\
GR & Graphene \\
HAADF & High-angle annular dark-field \\
HMT & Hexamethylenetetramine \\
ISO & International Organization for Standards \\
ITO & Indium tin oxide \\
LED & Light emitting diodes \\
LSPR & Localized surface plasmon resonance \\
NPs & Nanoparticles \\
NRs & Nanorods \\
PL & Photoluminescence \\
PPC & Persistent photoconductivity \\
SEM & Scanning electron microscopy \\
SiC & Silicon carbide \\
STEM & Scanning transmission electron microscopy \\
SUT & Sample under test \\
TCO & Transparent conductive oxide \\
TEM & Transmission electron microscopy \\
UHV & Ultra-high vacuum \\
XPS & X-ray photoelectron spectroscopy \\
XRD & X-ray diffraction \\
ZnO & Zinc oxide \\
ZnO:Al & Al-doped ZnO \\
ZnO-NPs & Zinc oxide nanoparticles \\
ZnO-NRs & Zinc oxide nanorods \\
& \\
\hline
\end{tabular}




\section{Table of Contents}

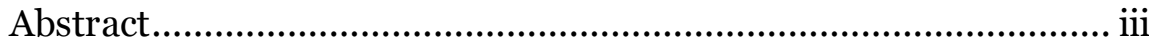

Acknowledgements ......................................................................

List of Papers …….........................................................................

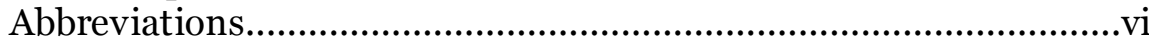

Table of Contents.........................................................................

1 Introduction …...................................................................... 9

1.1 Heterostructures and nanocomposites ............................... 9

1.2 Graphene/semiconductor heterojunctions........................10

1.3 Aim and Outline of this Thesis........................................... 11

2 Graphene-zinc oxide nanocomposites ....................................13

$2.1 \quad$ Materials background ......................................................13

2.1.1 Graphene ..............................................................13

2.1.2 Zinc oxide and doping ...........................................14

2.1.3 GNP/ZnO-NRs nanocomposites ............................15

2.1.4 Silver iodide (AgI) semiconductor .........................15

$2.2 \mathrm{GNP} / \mathrm{ZnO} \mathrm{O}$-NRs nanocomposite synthesis ..........................16

2.2.1 Seeding layer and $\mathrm{ZnO}$-NPs growth .....................16

2.2.2 Hydrothermal growth of $\mathrm{ZnO}-\mathrm{NRs}$.......................17

2.2.3 Aluminum doping in $\mathrm{ZnO}$.....................................17

$2.3 \mathrm{ZnO} / \mathrm{GNP} / \mathrm{Ag} / \mathrm{AgI}$ nanocomposite synthesis ....................18

$3 \quad$ Electronics applications .........................................................19

3.1 Effects of $\mathrm{pH}$ on the morphology of the $\mathrm{ZnO}$-NRs..............19

3.2 Improving the conductivity of $\mathrm{ZnO}$-NRs by Al-doping..... 20

3.2.1 Al-doping in GNP/ZnO-NRs.................................21

3.2.2 Electrical resistivity of the nanocomposites..........21

3.3 Quality of bonds between GNPs and ZnO-NRs ................. 23

3.3.1 UV-Vis absorption spectroscopy............................23

3.3.2 Photoluminescence spectroscopy...........................25

3.4 Photoconductivity and charge separation efficiency ........ 28

$4 \quad$ Photocatalytic applications ..................................................... 33

4.1 Characterization of $\mathrm{ZnO} / \mathrm{GNP} / \mathrm{Ag} / \mathrm{AgI}$ nanocomposites.. 33

4.2 Elemental bonding and chemical shifts in XPS ................ 35

4.2.1 XPS background...............................................36

4.2.2 Core-level binding energy and chemical shift......38

4.2.3 XPS analysis of the $\mathrm{ZnO} / \mathrm{GNP} / \mathrm{Ag} / \mathrm{AgI}$ samples..38 
4.3 Photocatalytic performance of the $\mathrm{ZnO} / \mathrm{GNP} / \mathrm{Ag} / \mathrm{AgI}$

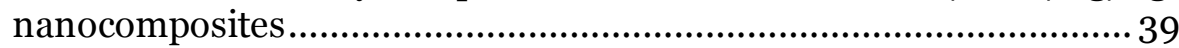
4.3.1 Photodegradation efficiency of CR dye ............... 39 4.3.2 Photocatalytic mechanism .................................... 40

Conclusions...................................................................................... 43

Outlook ….................................................................................. 44

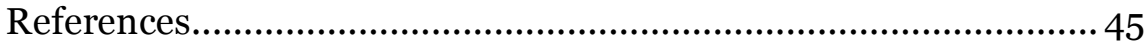

Part II: PAPERS ....................................................................... 51 


\section{Introduction}

The future of electro-optical devices relies on the development of new functional materials. Such materials can be produced by e.g. chemical conjugation of suitable elements, or by forming composites of existing materials. In fact, electronically graded composites with unprecedented electrical and optical properties can be designed and realized. Composite structures are multiphased materials merged together in such a way that the desired properties are obtained. A composite is designated as a nanocomposite if one of the phases has at least one dimension of less than $100 \mathrm{~nm}$, or a nanoscale repeated pattern of the phase structures.

\subsection{Heterostructures and nanocomposites}

A junction forms at the interface between two materials, brought physically into contact with each other. Depending on the similarity of the two materials with respect to e.g. crystal structure and doping type/level of the materials, different scenarios can occur. If the materials are similar, a homojunction forms at the interface. A p-n junction is one example of a homojunction formed in a semiconductor at the physical interface between two regions doped with donors and acceptors, respectively.

When two dissimilar semiconductor materials are bonded, a heterojunction is formed at the interface. Due to the difference in bandgap energy and the actual band alignments of the two semiconductors, many interesting devices such as laser diodes, light emitting diodes (LED), resonant tunneling diodes, photodetectors and solar cells, can be realized.

Heterojunctions are usually made by epitaxial growth of fairly lattice-matched semiconductors on top of each other. The critical parameter for epitaxial growth is matching of the lattice constants. Any existing mismatch in lattice constants induces atomic displacements (strain) and dislocations at the interface unless the epitaxial layer is very thin.

It has been shown that $1 \mathrm{D}$ structures e.g. nanorods (NRs), can tolerate significantly larger lattice mismatch by relieving the strain energy via elastic relaxation [1]. The stability of the structure depends on the lattice mismatch factor and the radius of the grown NRs (Fig. 1). This is 
one of the reasons why nanoparticles (NPs), often called oD structures, and NRs have been used in our research.

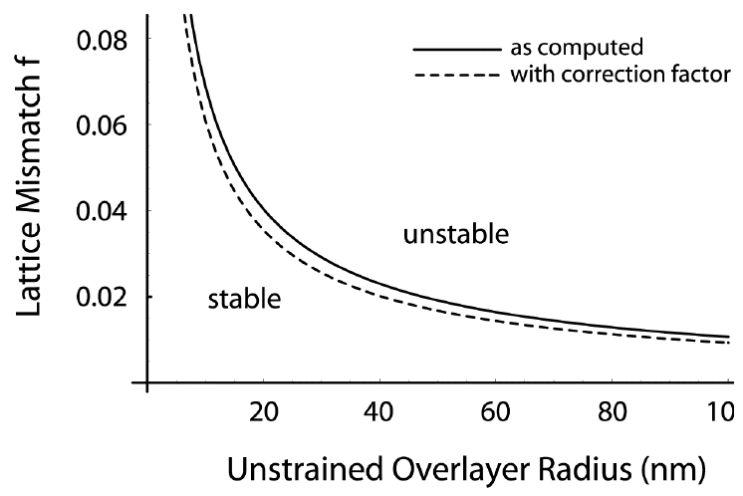

Fig. 1. Stability diagram for a heterostructure NR system with respect to formation of misfit dislocations [1]. (Used with permission)

\subsection{Graphene/semiconductor heterojunctions}

Although graphene (GR) has been widely studied more than a decade for various applications, graphene/semiconductor (GR/S) junctions have been investigated only in the few recent years. Graphene can be conjugated with a range of semiconductors for either enhancing the semiconductor performance, adding extra functionalities or increasing the overall efficiencies of optoelectronic and electrochemical devices. Various nanocomposites of GR and semiconductors, including group IV (Si), group II-VI ( $\mathrm{ZnO}, \mathrm{ZnS})$, group III-V ( $\mathrm{GaAs}, \mathrm{GaN})$ and metal oxide semiconductors $\left(\mathrm{TiO}_{2}, \mathrm{ITO}\right)$, have so far been realized [2].

Similar to the conventional metal/semiconductor junctions, GR/S junctions generally show rectifying characteristics and behave as a Schottky junction [3]. Schottky junctions are key devices in e.g. solar cells, photodetectors, MESFETs, RF components, Zener diodes, sensing applications and fast switching components in digital logic circuits. However, despite of the constant Fermi level in metals, the graphene Fermi level can easily be shifted due to its limited density of states close to the neutrality point [3]. This can result in potentially low resistance ohmic GR/S junctions, depending on doping and energy band alignment [2].

In addition to the strong covalent bonding in epitaxial heterostructures, van der Waals forces between different 2D materials can also form stable heterostructures with intriguing electronic properties. In fact, a van der Waals heterojunction can be formed between a 2D material and any passivated, dangling-bond-free material by only van der 
Waals interactions [4]. Using this interaction, any 2D material can in principle be integrated with material structures of any dimensionality, forming $2 \mathrm{D}-\mathrm{oD}, 2 \mathrm{D}-1 \mathrm{D}$ and $2 \mathrm{D}-3 \mathrm{D}$ heterostructures.

Opposite to conventional epitaxial heterostructures, lattice matching is not important in the above-mentioned 2D heterostructures. Because of the discontinuity in the band structure, tunneling and hopping are the dominant charge transfer mechanisms, causing additional junction resistance at the interface [4].

\subsection{Aim and Outline of this Thesis}

The aim of this thesis is to develop new nanocomposite materials based on GR nanoplates for electronic and photocatalysis applications. The available materials for conductive printed electronics suffer from low conductivity and high costs. Also, the efficiency of photocatalysts is limited by poor absorption of light and high recombination rates of the photogenerated carriers.

Forming composites of available materials is a promising way to produce new functional materials with improved electrical and optical properties. Two or more different solid materials with unique properties can be merged into a new composite with enhanced performance.

This thesis consists of two parts. In the first part, in Chapter 2, the necessary background of the basic materials and the nanocomposites is given. In addition, the synthesis procedure is discussed.

In Chapter 3, the GNP/ZnO-NRs nanocomposites for electronics applications are discussed. The effect of $\mathrm{pH}$ on the morphology of $\mathrm{ZnO}-$ NRs and the conductivity of the corresponding nanocomposite is studied. The bonding quality between the GNPs and $\mathrm{ZnO}-\mathrm{NRs}$ is examined by optical characterization. A short introduction to UV-Vis spectroscopy and photoluminescence spectroscopy (PL) is also given. The charge separation mechanisms and persistent photoconductivity (PPC) observed in the nanocomposites are investigated in more details.

Chapter 4 deals with the photocatalysis application by incorporating a new plasmonic semiconductor, silver iodide (AgI) nanoparticles, with the GNP/ZnO nanocomposite. The added AgI will absorb light in the visible range, resulting in an enhancement of the photocatalytic efficiency under solar light irradiation. X-ray photoelectron spectroscopy (XPS) was employed to study the synthesized samples. A detailed background to XPS analysis is also given in this chapter, which will help to better understand the results. Moreover, the photocatalytic efficiency of different samples is compared and the novel proposed photocatalytic mechanism is discussed at length. 
The second part of this thesis comprises the results of the scientific research, published in two papers. Paper I describes the GNP/ZnO-NRs nanocomposites, developed for electronics applications. The background and results of Paper I are discussed in Chapter 3, while Paper II (submitted) is more connected to Chapter 4, dealing with the results of a photocatalysis study of $\mathrm{ZnO} / \mathrm{GNP} / \mathrm{Ag} / \mathrm{AgI}$ nanocomposites. 


\section{Graphene-zinc oxide nanocomposites}

The heart of this work is based on composites of graphene and $\mathrm{ZnO}$ and their electro-optical and photochemical properties. Two different main types of nanocomposites were synthesized and studied, including; GNP/ZnO-NRs (doped and undoped), and ZnO-NPs/GNP/AgI. In the first series of the nanocomposites, graphene is the dominating material. They mainly developed for electronics application. The next series have been specifically optimized for photocatalysis application and the $\mathrm{ZnO}-$ NPs are dominated for this purpose. In this chapter, an introductory review of the used materials is given and then the nanocomposite synthesis methods will be discussed. The characterization and the application of each nanocomposite will be considered in the next chapters.

\subsection{Materials background}

\subsubsection{Graphene}

Graphene (GR), a 2D honeycomb lattice structure, is composed of a single layer of carbon atoms. It is one of the most widely studied materials for the last 15 years due to its remarkable properties e.g. excellent carrier mobility (up to $10^{6} \mathrm{~cm}^{2} \mathrm{~V}^{-1} \mathrm{~s}^{-1}$ ), low electrical resistivity (0.1-6 $\mathrm{k} \Omega / \square$ for a single layer with $97.7 \%$ optical transparency), chemical stability and mechanical strength (double to that of CNTs) [5]. The special properties of graphene stem from the three hybridized $\mathrm{sp}^{2}$ orbitals with $\sigma$-bonds between carbon atoms and out-of-plane $\pi$-bonds. By increasing the number of graphene layers, its properties changes dramatically into those of graphite. As a common arbitrary threshold, backed up by the International Organization for Standards (ISO), more than ten graphene layers is identified as graphite instead of graphene [6]. According to this standard, graphene as a single layer abbreviated as $1 \mathrm{LG}$, bilayer graphene as 2LG and few-layered graphene as FLG.

Several different graphene synthesis techniques have been introduced and categorized into three main classes; i) CVD-grown sheets, ii) epitaxial growth on SiC surfaces and iii) exfoliation flakes [5,7]. CVD and epitaxial growth are effective bottom-up, high quality, but more expensive wafer scale approaches that can produce single and multiple layer graphene films for carbon-based electronics. 
The other approach is a top-down, simple, high volume production and low-cost technique based on exfoliation of bulk graphite into graphene nanoplates (GNP). In the chemical exfoliation technique, chemicals are intercalated between the graphite layers which weakens the cohesive van der Waals forces, causing graphite expansion and finally exfoliation $[8,9]$. The finally produced GNPs by the exfoliation technique contains flakes with a range of different thicknesses and lateral sizes that can be dispersed in a liquid or be in a powder form. In practice, a significant amount of flakes can be found, having excess layers than FLG due to agglomeration between the particles. In all of this work, we have used GNP, synthesized by the chemical exfoliation technique.

\subsubsection{Zinc oxide and doping}

Zinc oxide is a well-known unintentionally n-type semiconductor with a hexagonal wurtzite type structure and a wide direct bandgap of 3.2-3.4 $\mathrm{eV}$ at room temperature. Thanks to the electronic properties of $\mathrm{ZnO}$, a wide range of applications from optoelectronic devices (e.g. LEDs and solar cells) to electrochemical applications and sensors have been realized and tested [10]. Moreover, $\mathrm{ZnO}$ can be grown in different nanostructured shapes e.g. nanoparticles, nanorods and thin films. Many different $\mathrm{ZnO}$ growth techniques such as sputtering, epitaxial growth, CVD and more, have successfully been utilized and verified [11]. In addition to these more complex techniques, hydrothermal growth has been demonstrated as a promising, inexpensive, lowtemperature $\left(<100{ }^{\circ} \mathrm{C}\right)$ solution-based procedure for $\mathrm{ZnO}$ synthesis. In this work, we have used low-temperature hydrothermal growth for all the synthesis procedures.

$\mathrm{ZnO}$ can also be doped with various elements to tune the electronic properties. Although n-type doping of $\mathrm{ZnO}$ using group 13 elements is simple, p-type doping of $\mathrm{ZnO}$ is notoriously difficult [10]. It has been shown that a doping level up to the degenerate level is achievable for $\mathrm{ZnO}$, which changes the electrical properties from those of an insulator to a metal [11]. Also, magnetic or ferromagnetic samples can be grown by doping $\mathrm{ZnO}$ with transition metal elements [10,11].

Aluminum is one of the most suitable $\mathrm{n}$-dopants in $\mathrm{ZnO}$. Unless the doping concentration is very high, the incorporated $\mathrm{Al}$ atoms replace $\mathrm{Zn}$ as a substitutional donor, which facilitates a tuning of the Fermi level to the degenerate metallic limit. In fact, $\mathrm{Al}$-doped $\mathrm{ZnO}$ (AZO) has been widely studied as a transparent conductive oxide (TCO), and is presently considered as one of the most promising candidates to substitute conventional TCOs on the market [12-15]. A comparison of different TCOs including, AZO can be found in Ref. [16]. 


\subsubsection{GNP/ZnO-NRs nanocomposites}

Even though graphene has many promising characteristics for electronic applications, it also has properties that are less desirable e.g. high hydrophobicity and a strong tendency not to interact with other materials. Moreover, the large van der Waals forces between the high aspectratio GNPs, results in undesired agglomeration into large particles. In order to use graphene in electronic or photocatalysis applications, it must therefore be efficiently dispersed in an appropriate medium. The most common way for dispersing GNPs is to use chemical surfactants to introduce static charges on the GNPs that keep them dispersed by electrostatic repulsion forces [17]. Unfortunately, this method deteriorates the electrical and optical properties of graphene.

The idea here is to form composites of GNPs and different $\mathrm{ZnO}$ structures to physically separate the nanoplates and prevent them from agglomeration. The $\mathrm{ZnO}$ structure can be NPs or NRs, depending on the application. In Chapter 3, GNP/ZnO-NRs is used for electronic applications, while in Chapter 4, $\mathrm{ZnO}-\mathrm{NP} / \mathrm{GNP}$ decorated with AgI NPs is utilized for photocatalysis applications.

It has been shown that $\mathrm{ZnO}$ can efficiently be grown on the GNPs surface, despite of their hydrophobicity [18-21]. The key parameter for growing heterostructures, is the lattice mismatch between the different materials. Graphene and $\mathrm{ZnO}$ have a relatively small lattice mismatch of about $2 \%[22,23]$ that allows the formation of a strong atomic bond between them [19]. From an electrical point-of-view, this fairly well matched interface between GNPs and $\mathrm{ZnO}$ can form a low-ohmic contact $[24,25]$, suitable for electrical applications.

\subsubsection{Silver iodide (AgI) semiconductor}

Silver-based semiconductors including silver oxide, silver sulfide and silver halides, particularly AgI, have attracted a lot of attention for photocatalysis applications due to their excellent visible light absorption [26]. These Ag-based materials are usually very light sensitive and easily decompose with visible light illumination. And this has made them as an extensively used material in photography for many years. In the last two decades, it has been shown that these materials can also be used as excellent photocatalysts [26].

This contradictory has been explained by a self-stabilizing mechanism where clusters of reduced silver atoms $\left(\mathrm{Ag}^{\circ}\right)$ are formed on the silver halide NPs at the initial time of illumination. Subsequently, these Ag-NPs trap the rest of the photogenerated electrons and thereby prevent further reduction of $\mathrm{Ag}^{+}$atoms. Moreover the $\mathrm{Ag}-\mathrm{NPs}$ act as local- 
ized surface plasmon resonators (LSPR) that lead to a strongly enhanced visible light absorption and photocatalysis efficiency [26].

AgI, with bandgap energy of $2.8 \mathrm{eV}$, can form composites with $\mathrm{ZnO}$ to shift the absorption peak into the visible range [27]. Also, it has been reported that incorporating AgI with graphene enhances the photocatalytic activity of pure AgI and improves the charge separation of photogenerated carriers under illumination [28]. In Chapter 4, we discuss $\mathrm{ZnO} / \mathrm{GNP} / \mathrm{Ag} / \mathrm{AgI}$ nanocomposites for solar-driven photocatalysis applications.

\subsection{GNP/ZnO-NRs nanocomposite synthesis}

As already mentioned, we used a hydrothermal solution-based procedure to grow ZnO-NRs on the surface of the GNPs. All the chemicals used in this work were purchased from Sigma Aldrich and used without any further purification, including the multilayer GR powder, zinc acetate dehydrate ${ }^{1}$, zinc nitrate hexahydrate ${ }^{2}$, hexamethylenetetramine $(\mathrm{HMT})^{3}$, potassium hydroxide $(\mathrm{KOH})$, silver nitrate $\left(\mathrm{AgNO}_{3}\right)$, and sodium iodide (NaI). Deionized (DI) water was used in all steps.

\subsubsection{Seeding layer and $\mathrm{ZnO}-\mathrm{NPs}$ growth}

To grow ZnO-NRs on the surface of GNPs, they first need to be seeded by a layer of ZnO-NPs. Although several reports have demonstrated that graphene itself is a good substrate candidate for $\mathrm{ZnO}$ growth [REF], seeding it with ZnO-NPs improves the growth quality and enhances the attachment between the GNPs and the ZnO-NRs. Moreover, ZnO-NPs can simultaneously grow on the GNPs during the ultrasonic dispersion of the solution. This can help to distribute ZnO-NPs between the layers of GNPs and better seeding coverage.

To seed the GNPs, a dispersion of GNPs in DI-water with a concentration of 0.5 g.l $\mathrm{l}^{-1}$, was prepared using an ultrasonic bath for $10 \mathrm{~min}$. Subsequently a solution of zinc acetate in water was mixed with the GNPs dispersion while stirring. The final concentration of zinc acetate in the solution was $5 \mathrm{mM}$. Afterwards, a dissolved $\mathrm{KOH}$ solution in water with a concentration of $25 \mathrm{mM}$ (in the final seed solution), was added dropwise to the seed solution at $60{ }^{\circ} \mathrm{C}$ in an ultrasonic bath and kept for 10 min to complete the seeding procedure.

Then the composite was washed in water and centrifuged (3000 rpm, $10 \mathrm{~min}$ ) three times and annealed in nitrogen at $300{ }^{\circ} \mathrm{C}$ for $30 \mathrm{~min}$.

\footnotetext{
${ }^{1} \mathrm{Zn}\left(\mathrm{CH}_{3} \mathrm{COO}\right)_{2} \cdot 2 \mathrm{H}_{2} \mathrm{O}$

${ }^{2} \mathrm{Zn}\left(\mathrm{NO}_{3}\right)_{2} \cdot 6 \mathrm{H}_{2} \mathrm{O}$

${ }^{3} \mathrm{C}_{6} \mathrm{H}_{12} \mathrm{~N}_{4}$
} 
This procedure improves the attachment between ZnO-NPs and the GNPs. Fig. 2 shows a scanning electron microscopy (SEM) image of GNPs decorated with $\mathrm{Al}$-doped $\mathrm{ZnO}-\mathrm{NPs}$. The process of $\mathrm{Al}$ doping will be discussed in section 2.2.3.

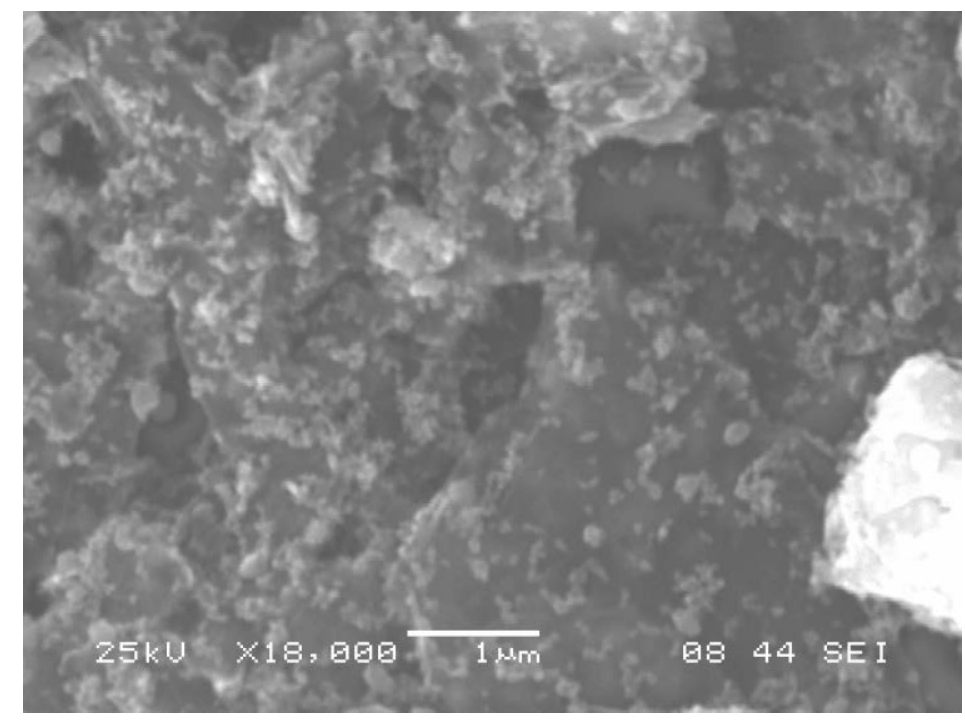

Fig. 2. SEM image of GNPs decorated with Al-doped ZnO-NPs.

\subsubsection{Hydrothermal growth of ZnO-NRs}

The seeded GNPs were now ready for $\mathrm{ZnO}$-NRs growth. To do that, two solutions of $25 \mathrm{mM}$ of zinc nitrate and $25 \mathrm{mM}$ HMT in water, were prepared separately and added together while stirring. The $\mathrm{pH}$ of the growth solution can be adjusted in the range from 6.5 to 11 by adding ammonia. In this work we studied the effect of $\mathrm{pH}$ (6.6 and 11) on the morphology of $\mathrm{ZnO}-\mathrm{NRs}$. Afterwards, the temperature of the growth solution was increased to $75{ }^{\circ} \mathrm{C}$ and the seeded GNPs were added under a mild stirring and kept for $2 \mathrm{~h}$ to complete the growth of $\mathrm{ZnO}-\mathrm{NRs}$. Subsequently the grown GNP/ZnO-NRs nanocomposite was washed in water and centrifuged (3000 rpm, $10 \mathrm{~min}$ ) for three times and dried in an oven at $90{ }^{\circ} \mathrm{C}$ for $2 \mathrm{~h}$.

The synthesized GNP/ZnO-NRs powder can be coated on a solid substrate by spin coating for further analysis or be deposited by a vacuum filtration method [17] to form a smooth thicker layer.

\subsubsection{Aluminum doping in $\mathrm{ZnO}$}

The $\mathrm{ZnO}-\mathrm{NRs}$ and $\mathrm{ZnO}-\mathrm{NPs}$ can be doped by $\mathrm{Al}$ during the hydrothermal growth process if a proper $\mathrm{Al}$ precursor is introduced into the 
growth solution. For the seeding layer, the precursor can simply be a piece of pure $\mathrm{Al}$ immersed into the seed solution. The high $\mathrm{pH}$ value of 13 of the seed solution will guarantee a partial dissolving of the $\mathrm{Al}$ piece, leading to the desired $\mathrm{Al}$ doping of the $\mathrm{ZnO}-\mathrm{NPs}$ [29].

To dope the $\mathrm{ZnO}-\mathrm{NRs}$ with $\mathrm{Al}$, a different strategy was employed by using aluminum nitrate nonahydrate ${ }^{4}$ as the $\mathrm{Al}$ precursor [29-31]. An aqueous solution of zinc nitrate with concentration of $2 \mathrm{mM}$ (in the final growth solution) was prepared and stirred overnight before adding to the growth solution to make sure that the $\mathrm{Al}$ is uniformly dissolved and dispersed. Subsequently, the prepared solution was added to the mixture of GNPs and growth solution as explained in the section above.

\section{3 $\mathrm{ZnO} / \mathrm{GNP} / \mathrm{Ag} / \mathrm{AgI}$ nanocomposite synthesis}

The $\mathrm{ZnO} / \mathrm{GNP} / \mathrm{Ag} / \mathrm{AgI}$ nanocomposites were prepared via an ultrasonic-assisted hydrothermal solution-based procedure. First, the $\mathrm{ZnO} / \mathrm{GNP}$ nanocomposite was prepared according to the hydrothermal seeding growth procedure explained in section 2.2.1, but with different concentrations. Concentrations of $10 \mathrm{mg} \mathrm{l}^{-1}, 10 \mathrm{mM}$ and $50 \mathrm{mM}$ of GNP, zinc acetate and $\mathrm{KOH}$, respectively, were chosen to achieve the final GNP-to-ZnO weight ratio of 1:99. The obtained $\mathrm{ZnO} / \mathrm{GNP}$ nanocomposite was then washed in water and acetone and centrifuged at $3000 \mathrm{rpm}$ three times for 10 minutes, followed by drying in an oven at $120{ }^{\circ} \mathrm{C}$ overnight.

Subsequently, Ag/AgI NPs were grown on the ZnO/GNP nanocomposite via an ultrasonic irradiation method. The prepared $\mathrm{ZnO} / \mathrm{GNP}$ nanocomposite was dispersed in DI-water and silver nitrate ${ }^{5}$ was added to the suspension, stirring for 30 minutes. Then, an aqueous solution of sodium iodide ${ }^{6}$ was added dropwise, followed by one hour of ultrasonication and two times washing and centrifugation. The synthesized nanocomposite finally dried in an oven at $75{ }^{\circ} \mathrm{C}$ for 6 hours. Three different weight ratios of $\mathrm{ZnO} / \mathrm{GNP}$ to $\mathrm{Ag} / \mathrm{AgI}$ of $\mathrm{X}=10 \%, 20 \%$ and $30 \%$ were prepared and denoted $\mathrm{ZnO} / \mathrm{GNP} / \mathrm{Ag} / \mathrm{AgI}(\mathrm{X})$. More details about the growth can be found in the Appendix of Paper II. In addition, pristine $\mathrm{ZnO}-\mathrm{NPs}$ and $\mathrm{ZnO} / \mathrm{Ag} / \mathrm{AgI}$ nanocomposites were also prepared as reference samples.

\footnotetext{
${ }_{5}^{4} \mathrm{Al}\left(\mathrm{NO}_{3}\right)_{3} \cdot 9 \mathrm{H}_{2} \mathrm{O}$

${ }_{5} \mathrm{AgNO}_{3}$

${ }^{6} \mathrm{NaI}$
} 


\section{Electronics applications}

The development of $3 \mathrm{D}$-printed electronics is growing very fast. Many electronic components and devices can be fabricated by additive manufacturing if highly conductive and inexpensive printable materials are available. Here in this chapter, we investigate the electrical and optical properties of our newly developed GNP/ZnO:Al-NRs nanocomposites, well-suited for printed electronics. The effects of two different morphologies of $\mathrm{ZnO}-\mathrm{NRs}$ with varying $\mathrm{Al}$ doping concentration on the nanocomposite conductivity are studied at length. In addition, optical analysis techniques such as absorption spectroscopy, photoluminescence (PL) and photoconductivity were conducted for better understanding of the behavior of the nanocomposites.

\subsection{Effects of $\mathrm{pH}$ on the morphology of the $\mathrm{ZnO}-\mathrm{NRs}$}

As prepared $\mathrm{ZnO}$-NRs growth solutions have a typical $\mathrm{pH}$ value of 6.6. Ammonia can be added to the solution to tune the $\mathrm{pH}$ up to around 11 . The transparency of the solution changes with the $\mathrm{pH}$ value. At a $\mathrm{pH}$ of 6.6 it is fully transparent. Upon increasing the $\mathrm{pH}$ value, the solution first becomes milky and opaque in the $\mathrm{pH}$ range of 7.8-10.8 and then it again turns transparent at a $\mathrm{pH}$ of 11 . The opaque window in the $\mathrm{pH}$ range indicates the presence of a precipitate of $\mathrm{ZnO}$ particles in a supersaturated solution. The $\mathrm{pH}$ values of 6.6 and 11 were selected to avoid this unstable condition in the solution.

Two morphologies of GNP/ZnO-NRs at $\mathrm{pH} 6.6$ and 11 were grown with and without $\mathrm{Al}$-doping. We found that as long as the concentration ratio of $\left[\mathrm{Al}\left(\mathrm{NO}_{3}\right)_{3}\right] /\left[\mathrm{Zn}\left(\mathrm{NO}_{3}\right)_{2}\right]$ was less than $8 \%$, Al-doping did not change the GNP/ZnO-NRs morphology significantly. This is in good agreement with Ref. [30,31]. Fig. 3 shows GNP/ZnO-NRs grown at a $\mathrm{pH}$ of 6.6 and 11.

ZnO-NRs grown at pH 6.6 (Fig. 3 (a)) are hexagonal, thicker (mean diameter $313 \mathrm{~nm}$ ), longer (1-2 $\mu \mathrm{m})$ and more sparse compared to $\mathrm{ZnO}$ NRs grown at higher $\mathrm{pH}$ values. The NRs grown at a $\mathrm{pH}$ of 11 are more

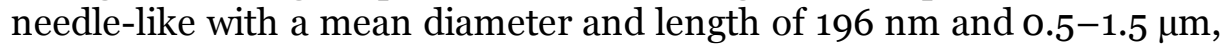
respectively (Fig. 3 (b)). The average density of $\mathrm{ZnO}-\mathrm{NRs}$ on the GNP 
surfaces was $1.4 \mu \mathrm{m}^{-2}$ for samples grown at $\mathrm{pH} 6.6$ and $2.4 \mu \mathrm{m}^{-2}$ for samples grown at $\mathrm{pH} 11$, as estimated from SEM images.
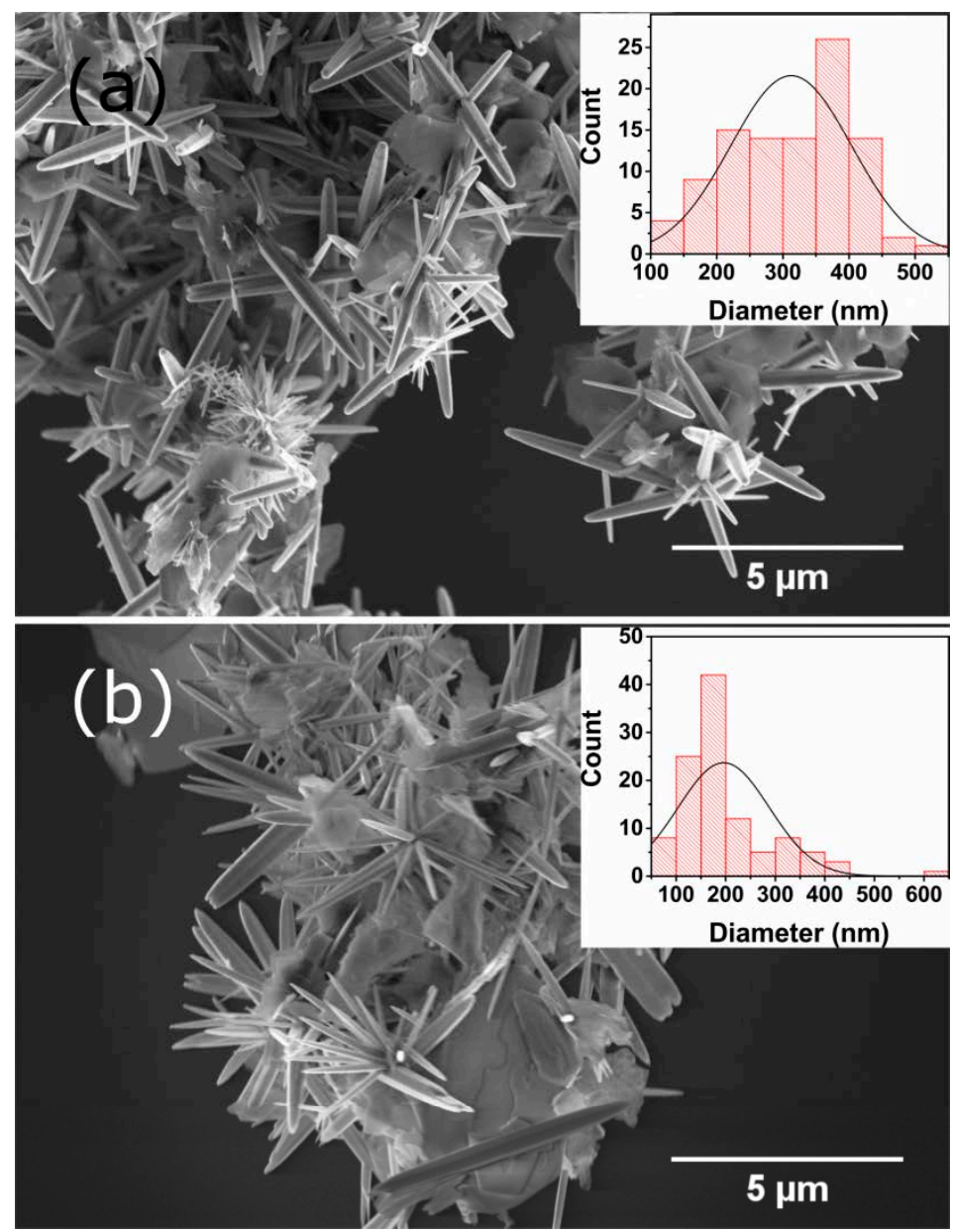

Fig. 3. SEM images of (a) thick hexagonal low-density ZnO-NRs grown at $\mathrm{pH}$ 6.6 and (b) thin needle-like, high-density $\mathrm{ZnO}-\mathrm{NRs}$ grown at $\mathrm{pH}$ 11. The insets show the size distribution of the NR diameter.

\subsection{Improving the conductivity of $\mathrm{ZnO}-\mathrm{NRs}$ by $\mathrm{Al}-$ doping}

The long-term goal of the efforts to realize highly conductive nanocomposites was to develop disruptive electronics by $3 \mathrm{D}$ printing. Due to the wide bandgap of $\mathrm{ZnO}$, it is expected that as-grown $\mathrm{ZnO}$-NRs will behave as an insulator. To increase the conductivity of the nanocomposites, we degenerately doped the $\mathrm{ZnO}-\mathrm{NRs}$ by $\mathrm{Al}$. 


\subsubsection{Al-doping in GNP/ZnO-NRs}

We used a constant $\mathrm{Al}$ concentration bath, with a ratio of $\left[\mathrm{Al}\left(\mathrm{NO}_{3}\right)_{3}\right] /\left[\mathrm{Zn}\left(\mathrm{NO}_{3}\right)_{2}\right]$ less than $8 \%$, for all the doped samples in order to maximize the conductivity without changing the $\mathrm{ZnO}$ morphology [30,31]. Fig. 4 shows energy dispersive x-ray spectroscopy (EDS) spectra revealing the abundance of different elements in the samples. Carbon (C) is the most abundant element observed in all composites with an atomic ratio of 6.5 to $\mathrm{Zn}$. The presence of $0.5^{-1.5}$ at\% $\mathrm{Al}$ is also detected in the doped composites.

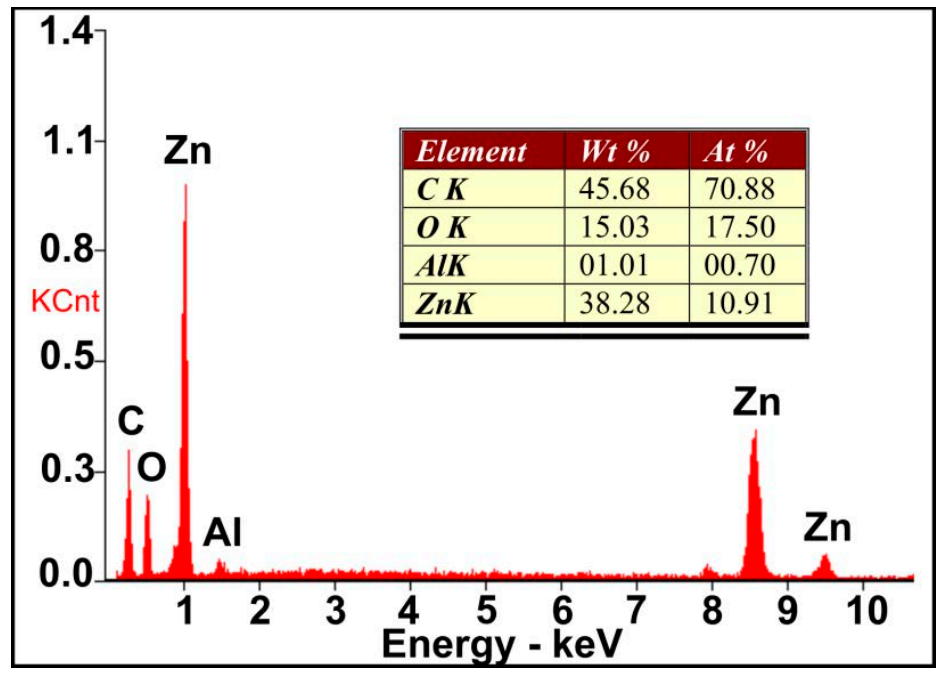

Fig. 4. EDS spectra for GNP/ZnO:Al-NRs grown at $\mathrm{pH} 11$.

A more accurate estimation of the doping concentration in the samples, based on the dopant concentration in the growth solution [32,33], lies in the range of $0.7-1.2$ at\%. The presence of oxygen (O) is almost 60 at\% higher than $\mathrm{Zn}$ in the samples. This excess of $\mathrm{O}$ could be related to the hydroxyl groups $\mathrm{OH}^{-}$and/or carboxyl groups $-\mathrm{COOH}$, or to adsorbed water on the GNPs or ZnO surfaces during the synthesis [21].

\subsubsection{Electrical resistivity of the nanocomposites}

The electrical resistivity of the nanocomposite samples was measured by a standard 4-point probe technique [34]. To do that, fairly thick layered materials must first be smoothly deposited. To deposit the randomized GNP/ZnO:Al-NRs microparticles, a vacuum filtration method [17] was employed. A water dispersion containing the microparticles was filtered by an Anodisc membrane filter from Whatman $(25 \mathrm{~mm}$ diameter, $0.2 \mu \mathrm{m}$ pore size) and dried at $70{ }^{\circ} \mathrm{C}$ in an oven. The thickness 
of the layers was measured by a contact profilometer and found to vary from 20 to $50 \mu \mathrm{m}$.

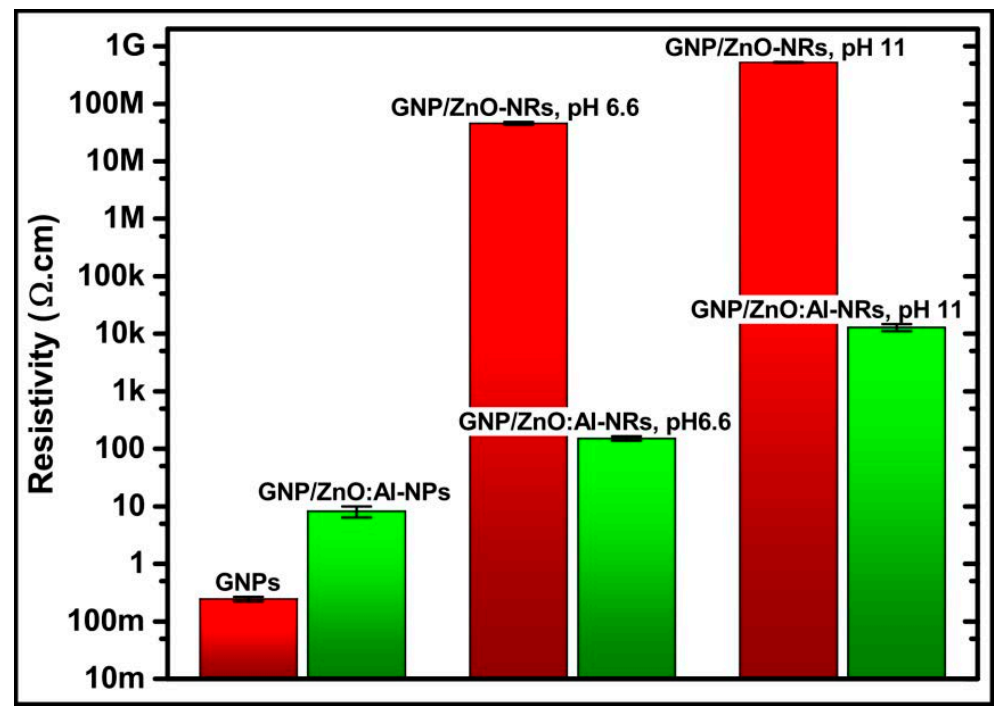

Fig. 5. Electrical resistivity of GNPs, GNP/ZnO:Al-NPs, GNP/ZnO-NRs (pH 6.6 and 11 ) and GNP/ZnO:Al-NRs (pH 6.6 and 11) measured by a 4-point probe method.

Fig. 5 shows a comparison of measured electrical resistivity for different samples. Evidently, pure GNPs show the lowest resistivity and it increases after decoration with ZnO:Al-NPs. This increase can be explained by the spacing introduced between the GNPs by the doped $\mathrm{ZnO}$ NPs. Since, GNPs are the most conductive particles in the nanocomposite, any separation between them leads to an increase in the resistivity. In addition, the chemical synthesis procedure can also modify the surface of the GNPs, which increases the resistivity.

Growth of undoped $\mathrm{ZnO}-\mathrm{NRs}$ on the decorated GNPs results in a further increase of the resistivity for both investigated morphologies, effectively converting the layers to insulators. This high resistivity shows that the GNPs are physically separated from each other by the non-intentionally doped $\mathrm{ZnO}-\mathrm{NRs}$. For the doped samples, the resistivity strongly decreased and the nanocomposites again became conductive due to the enhanced conductivity of the degenerately doped ZnO:Al-NRs. This shows that the NRs are indeed critical current paths, bridging over between different GNPs in the complex electrical network of the nanocomposite.

The $\mathrm{ZnO}-\mathrm{NRs}$ grown at $\mathrm{pH} 11$ show a comparably higher resistivity than those grown at $\mathrm{pH}$ 6.6. This suggests that GNPs coated with nano- 
needles are better dispersed. In the next chapters we testify this hypothesis by further optical characterization.

\subsection{Quality of bonds between GNPs and ZnO-NRs}

One important aspect of our developed nanocomposites is the bonding quality between the grown $\mathrm{ZnO}-\mathrm{NRs}$ and the GNPs. It can directly affect the composite conductivity and also optical properties. For further investigation, UV-Vis absorption and PL spectroscopy were employed.

\subsubsection{UV-Vis absorption spectroscopy}

UV-Vis spectroscopy probes the light intensity transmitted through or reflected from a medium at each wavelength. Fig. 6 shows a schematic of the UV-Vis spectrometer system (PerkinElmer Lambda 900) used in this work. A light beam from a deuterium lamp or a tungsten lamp, depending on the investigated wavelength region, is dispersed by the grating in the spectrometer and further split into two beams. One beam passes through a reference medium, while the other beam passes through the sample under test (SUT). The transmitted light is finally detected by a PbS or PMT photodetector (depending on the wavelength). To eliminate the effect of any optical component on the intensity in either of the beam paths, the system is initially calibrated by using the reference medium as SUT. Subsequently, the transmittance (T), or the absorption (A), is calculated based on the detected beam intensity passed through SUT and the reference (I and $\mathrm{I}_{\mathrm{O}}$ respectively), according to the Eq. (3.1) and Eq. (3.2). These equations are valid only if there is no reflection in the system. In practice, the reflection also should be taken into account otherwise; the A will only be an approximation.

$$
\begin{aligned}
& T=\frac{I}{I_{0}} \\
& A=-\log T
\end{aligned}
$$




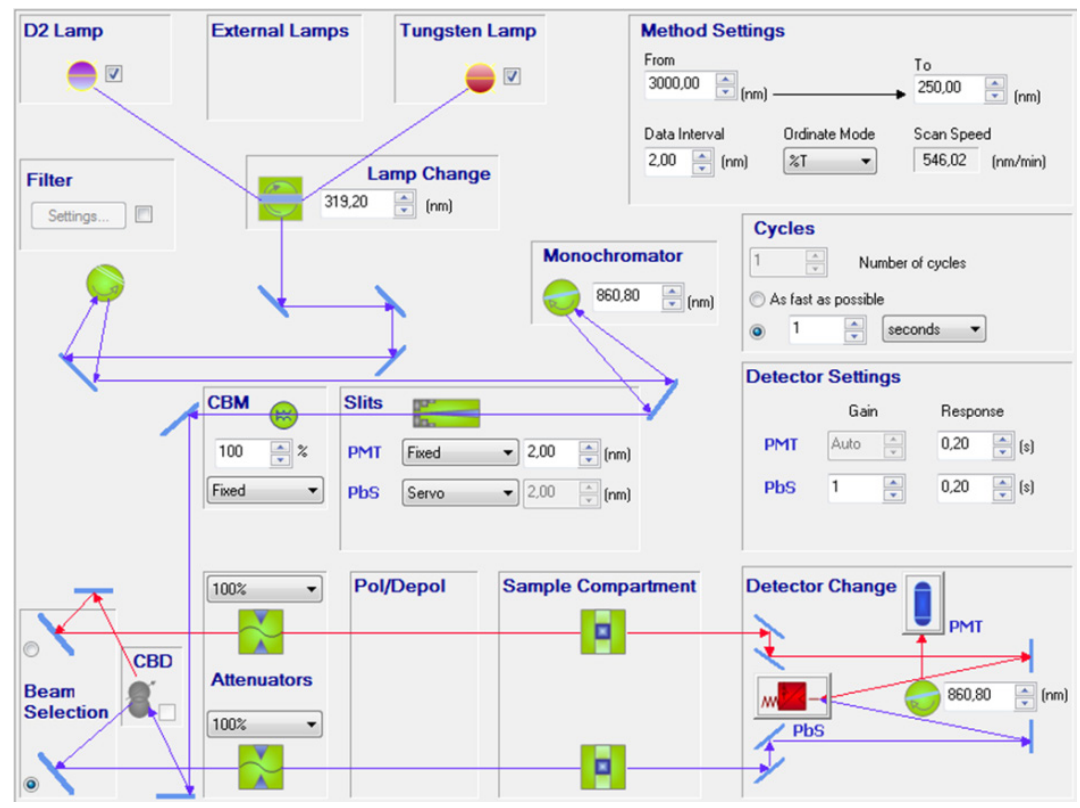

Fig. 6. Schematic of the UV-Vis spectrometer model PerkinElmer Lambda 900.

The two morphologies of $\mathrm{ZnO}-\mathrm{NRs}$, grown at different $\mathrm{pH}$, show different bonding quality. If the ZnO-NRs are weakly attached to the GNPs, or not attached at all, two phases (separated GNPs and ZnO-NRs) will be present in the dispersion. To compare the dispersity of the two morphologies, and the attachment of ZnO-NRs to the GNPs, timedependent UV-Vis spectroscopy was employed.

Three dispersions of GNPs and GNP/ZnO:Al-NRs grown at $\mathrm{pH} 6.6$ and 11 in isopropanol, respectively, were prepared. The timedependent absorption spectra of these dispersions were measured after every $5 \mathrm{~min}$ and after $24 \mathrm{~h}$. The absorption spectra of pure GNPs exhibit a rather flat characteristics dominated by spectral features beyond 1500 $\mathrm{nm}$ related to hydroxyl and/or carboxyl groups on the GNP surfaces (Fig. 7(a)). Fig. 7(b) and (c) display a strong absorption peak at about $380 \mathrm{~nm}$ for dispersions of GNP/ZnO:Al-NRs due to the ZnO bandgap.

The dispersions of GNPs and GNP/ZnO:Al-NRs grown at $\mathrm{pH} 11$ show a uniform decrease in absorption with time revolution due to particle precipitates. In contrast, Fig. $7(\mathrm{~b})$ shows that the absorption in GNP/ZnO:Al-NRs grown at a $\mathrm{pH} 6.6$ changes non-uniformly with wavelength. The long wavelength absorption response in our dispersions is dominated by the GNPs, while it relates to $\mathrm{ZnO}$ at short wavelengths. The non-uniform decrease in absorption observed in Fig. $7(\mathrm{~b})$ indicates the presence of two phases (separated GNPs and ZnO:Al-NRs) in the dispersion. The ZnO:Al-NRs settle faster than the GNPs. In con- 
trast, GNP/ZnO:Al-NRs grown at a $\mathrm{pH} 11$ forms a single phase in the dispersion, as evident from the uniform settling rate in Fig. $7(\mathrm{c})$. This implies that ZnO:Al-NRs are better attached to GNPs in high $\mathrm{pH}$ growth solutions.
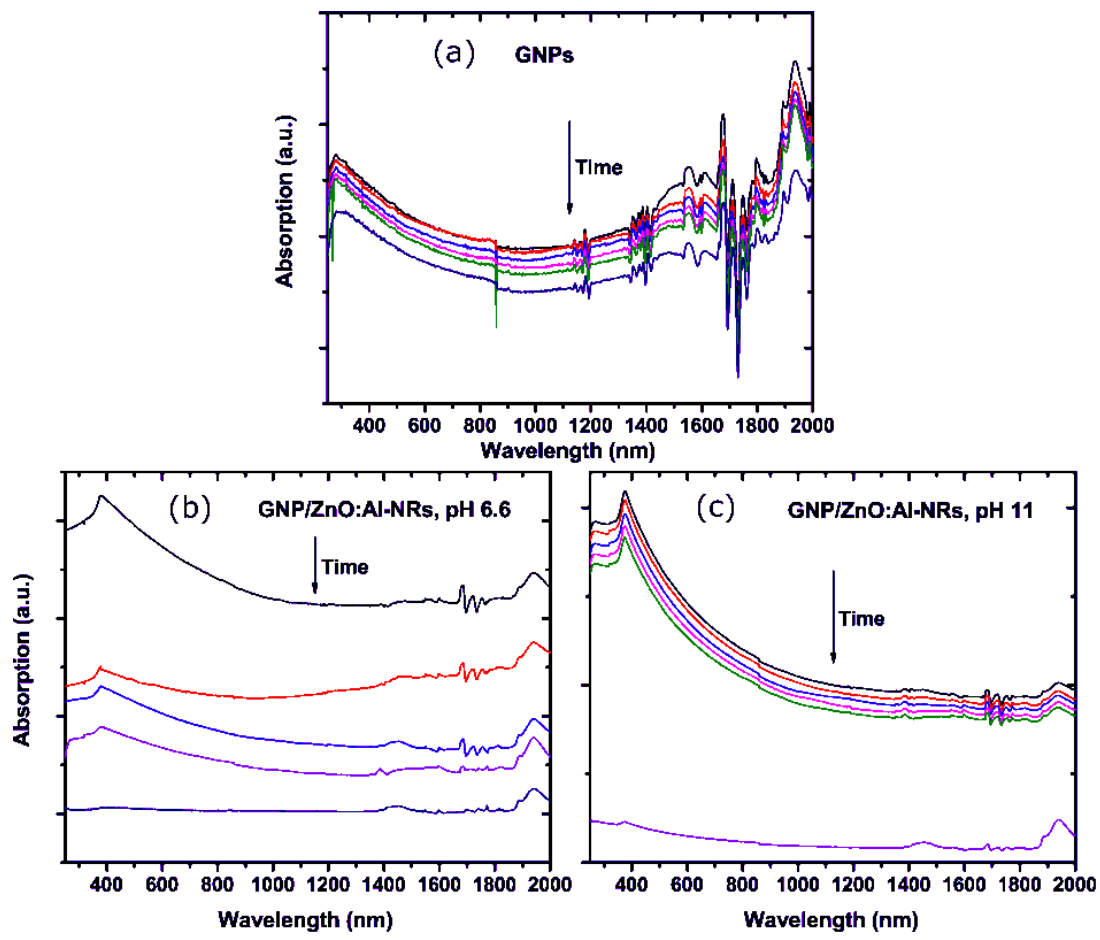

Fig. 7. Time-dependent UV-Vis spectroscopy. Temporal evolution of the absorption spectra after $0,5,10,15,20 \mathrm{~min}$ and $24 \mathrm{~h}$, respectively, for the dispersion of (a) GNPs, (b) GNP/ZnO:Al-NRs grown at pH 6.6 and (c) GNP/ZnO:AlNRs grown at $\mathrm{pH} 11$ in isopropanol. (Figure (a) has a different vertical scale)

\subsubsection{Photoluminescence spectroscopy}

Luminescence is the spontaneous emission of light resulting from other forms of excitation than heat (cold-body emission). The excitation energy can come from impinging electrons, electric current or absorbed photons with assigned names of cathodoluminescence, electroluminescence and photoluminescence, respectively. In contrast, light emitted due to heat is referred to as incandescence. 


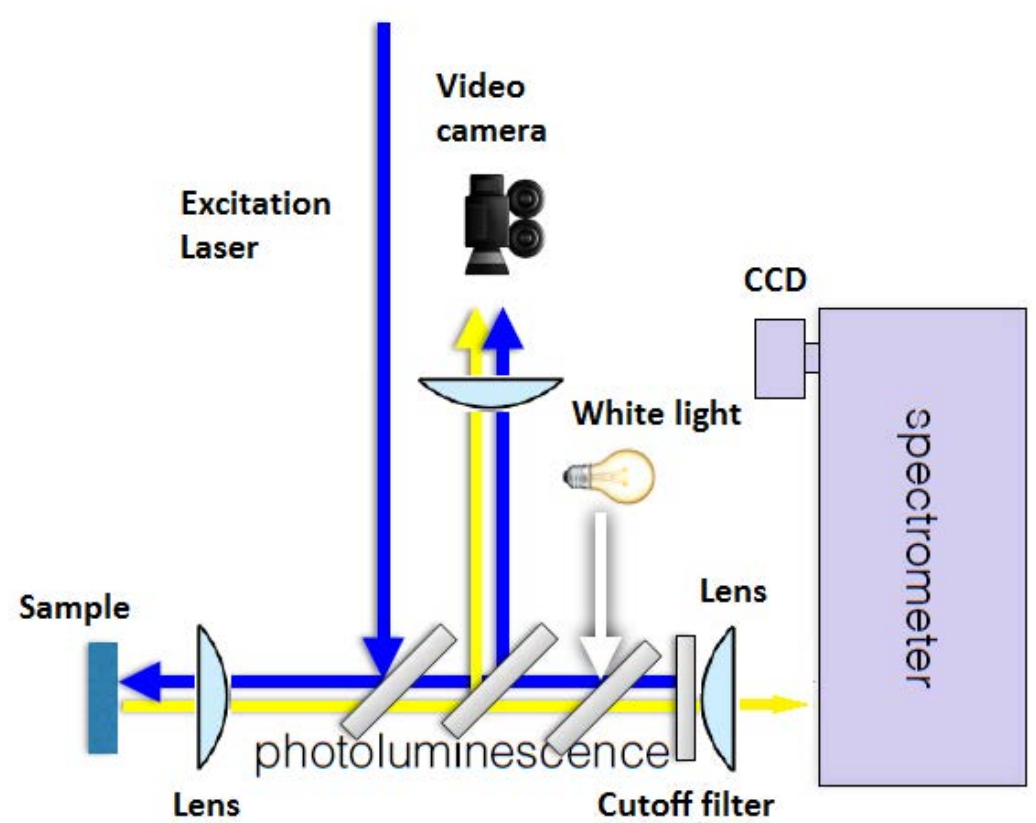

Fig. 8. Schematic of our room-temperature micro-PL setup

In this work, we used a room-temperature micro-PL ( $\mu$-PL) setup, including an $80 \mu \mathrm{W}, 266 \mathrm{~nm}$ excitation laser, schematically shown in Fig. 8 . The photon energy of the laser $(4.66 \mathrm{eV})$ was selected higher that the $\mathrm{ZnO}$ bandgap energy $(3.4 \mathrm{eV})$ to promote excitation of electrons from the valence band to the conduction band. The excited electron-hole pairs can radiatively recombine through the two main paths; i) nearbandedge emission (NBE) and ii) deep-level emission (DLE), consisting of conduction band to acceptor and donor to valence band recombination processes [35].

The main peaks in Fig. 9 show the ZnO NBE emission at 3.25-3.38 $\mathrm{eV}$ in agreement with the absorption measurements. The broad peak at $2.4 \mathrm{eV}$ indicates the visible light emission via deep levels in $\mathrm{ZnO}$ caused by zinc and oxygen vacancies [36]. Two distinct changes are readily observed in the $\mu$-PL spectra after doping the $\mathrm{ZnO}-\mathrm{NRs}$ with $\mathrm{Al}$; first, the DLE is suppressed due to filling of the defects levels by electrons (Al is a donor in $\mathrm{ZnO}$ ). This observation has previously been reported for Ga-doped ZnO-NRs [37]. Second, a blue-shift in the band-edge emission is observed which we attribute to the Burstein-Moss effect $[33,38,39]$. 

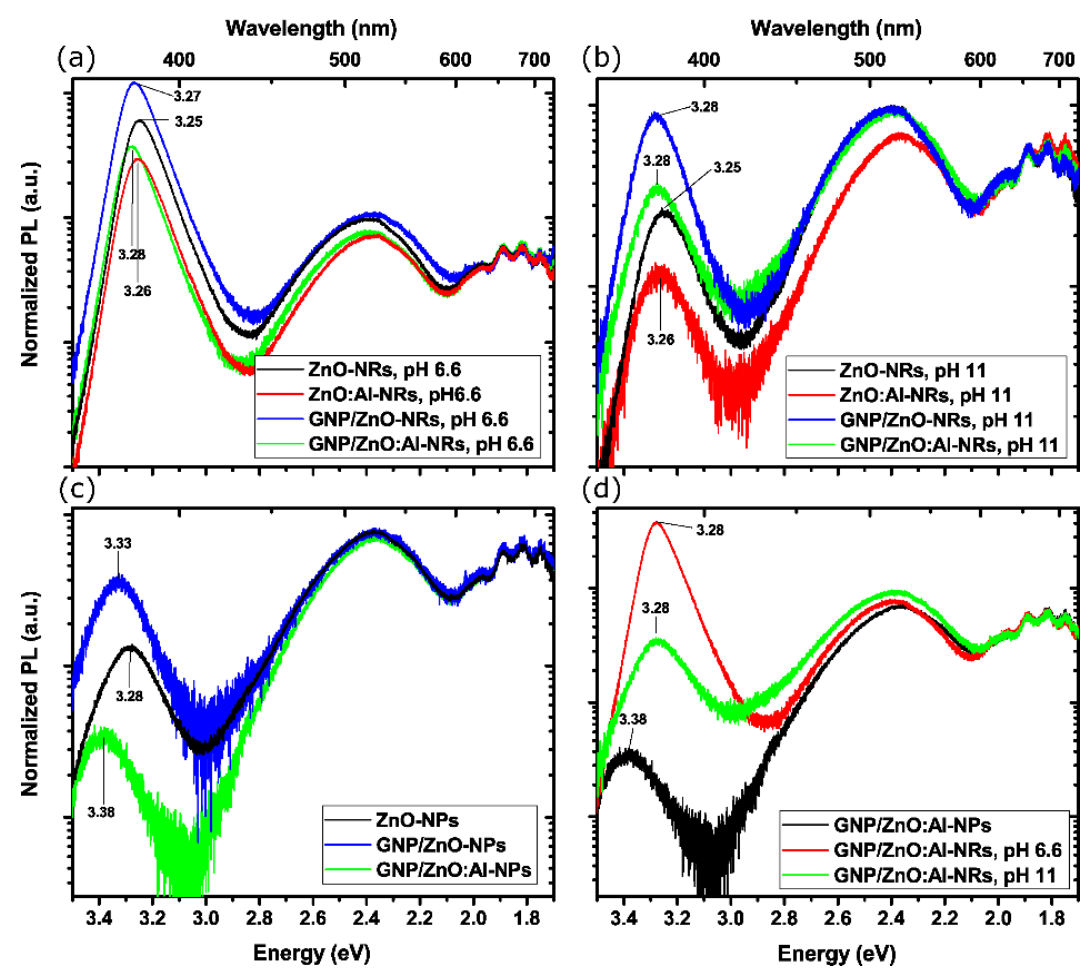

Fig. 9. Room-temperature $\mu$-PL spectra for different $\mathrm{ZnO}-\mathrm{NRs}$ nanocomposites grown at (a) $\mathrm{pH}$ 6.6, (b) $\mathrm{pH}$ 11, and (c) different GNP/ZnO-NPs nanocomposites. (d) Comparaative PL spectra for $\mathrm{GNP} / \mathrm{ZnO}$ :Al nanocomposites for three different $\mathrm{ZnO}$ morphologies.

Furthermore, the NBE is also suppressed by Al-doping. The ratio of NBE-to-DLE integrated intensities $\left(\mathrm{I}_{\mathrm{NBE}} / \mathrm{I}_{\mathrm{DLE}}\right)$ for different samples are summarized in Table 1 . In all the samples the $\mathrm{I}_{\mathrm{NBE}} / \mathrm{I}_{\mathrm{DLE}}$ ratio is decreased by adding $\mathrm{Al}$ to the composite. This decrease in UV-to-visible emission ratio can be explained by introducing impurity levels in the bandgap related to the doped $\mathrm{Al}$ ions and to a lower crystalline quality of the $\mathrm{ZnO}[38,40,41]$. More detailed explanations can be found in our Paper I [42]

Table 1. The NBE-to-DLE integrated intensity ratio $\left(\mathrm{I}_{\mathrm{NBE}} / \mathrm{I}_{\mathrm{DLE}}\right)$ for different ZnO-NRs composites.

\begin{tabular}{lcc}
\hline & Grown at pH 6.6 & Grown at pH 11 \\
\hline ZnO-NRs & 2.46 & 0.15 \\
ZnO:Al-NRs & 1.78 & 0.11 \\
GNP/ZnO-NRs & 4.25 & 0.46 \\
GNP/ZnO:Al-NRs & 1.85 & 0.21 \\
\hline
\end{tabular}


Comparing the $\mu$-PL for the two ZnO-NRs morphologies in Fig. 9(d) shows that thicker $\mathrm{ZnO}$-NRs grown at $\mathrm{pH} 6.6$ display a stronger bandedge PL emission than the thinner needle-like $\mathrm{ZnO}-\mathrm{NRs}$ grown at $\mathrm{pH} 11$ due to a lower surface-to-volume ratio. A high surface defect concentration on the thinner Zn-NRs not only causes a lower band-edge emission in the optical characteristics, but also leads to a higher electrical contact resistance (Fig. 5).

\subsection{Photoconductivity and charge separation efficiency}

A significant persistent photoconductivity (PPC) in $\mathrm{ZnO}$ has been demonstrated in many articles [43-46]. The PPC observed in $\mathrm{ZnO}$ is attributed to a spatial charge separation mechanism induced by the built-in electric field caused by a surface space-charge layer [47]. More details about the charge separation mechanism, have been discussed in the paper 1 [42].

The PPC was measured using a pulsed monochromatic optical excitation at room-temperature. A bias of $1 \mathrm{~V}$ was applied to a layer of the nanocomposite material, sandwiched between two ITO electrodes. The current was recorded under optical excitation with different wavelengths from 320 to $400 \mathrm{~nm}$.

As shown in Fig. 10, there is no detected photoresponse for wavelengths longer than the corresponding bandgap of $\mathrm{ZnO}$. Also, no PPC was observed in any of the highly Al-doped samples, not even under $254 \mathrm{~nm}$ illumination. This absence of PPC in highly doped samples can be explained by electrostatic screening of the surface oxygen ions that prevents holes from migrating towards the surface [47].

To compare the effect of nanocomposite morphology on the lifetime of the photogenerated electrons, time-dependent PPC measurements were pursued, and the corresponding excess electron life time $\left(\tau_{d}\right)$ was calculated. A typical rise and decay curve for the PPC (current) is shown in Fig. 11(a). 


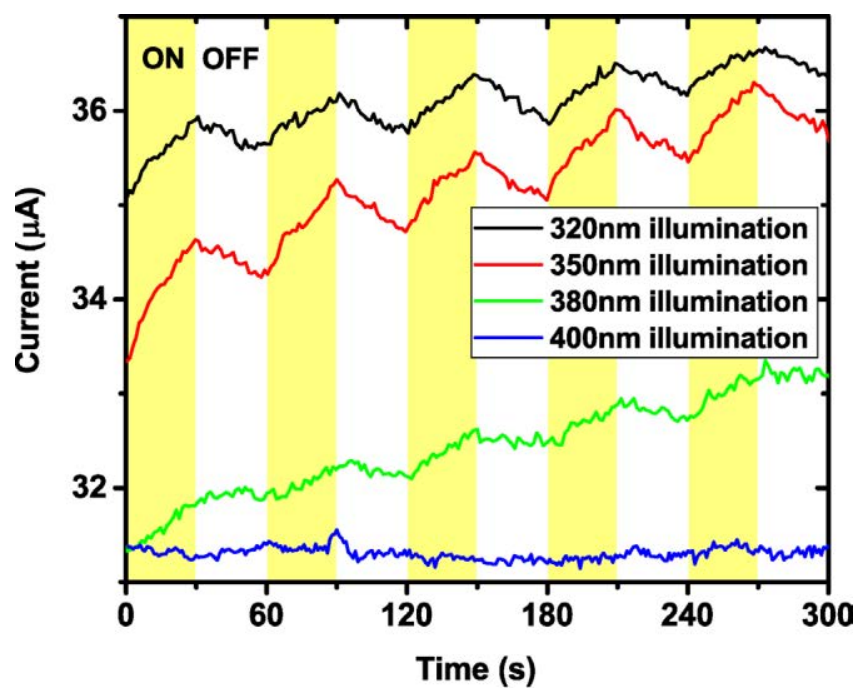

Fig. 10. The cycled PPC response for a GNP/ZnO-NRs sample at roomtemperature and $1 \mathrm{~V}$ applied bias.

Chemisorpted oxygen at the surface traps photogenerated holes, leaving behind an increasing density of mobile electrons in the conduction band, which results in a PPC after switching off the light. The PPC slowly decays due to recombination of photogenerated electrons with the holes trapped by the oxygen.

Fig. 11(b) shows the time-dependence of the PPC of undoped GNP/ZnO-NR samples with different morphology after $350 \mathrm{~nm}$ excitation. During the decay, the PPC is proportional to the density of electrons in the conduction band which in general decays exponentially with time [48]. The photoconductivity $\sigma_{\mathrm{ph}}$ (after subtracting the dark conductivity) decays exponentially according to:

$$
\begin{aligned}
& \frac{d \sigma_{p h}}{d t}=-\frac{\sigma_{p h}}{\tau_{d}}, \\
& \ln \sigma_{p h}=-\frac{t}{\tau_{d}}+\ln \sigma_{0}
\end{aligned}
$$

Here, $\tau_{d}$ is the decay time constant (excess electron lifetime) and $\sigma_{0}$ is the observed PPC directly after switching off the light. The extracted electron lifetime for samples with different morphology is calculated by a linear fitting to Eq. (3.4) and reported in Table 2. 

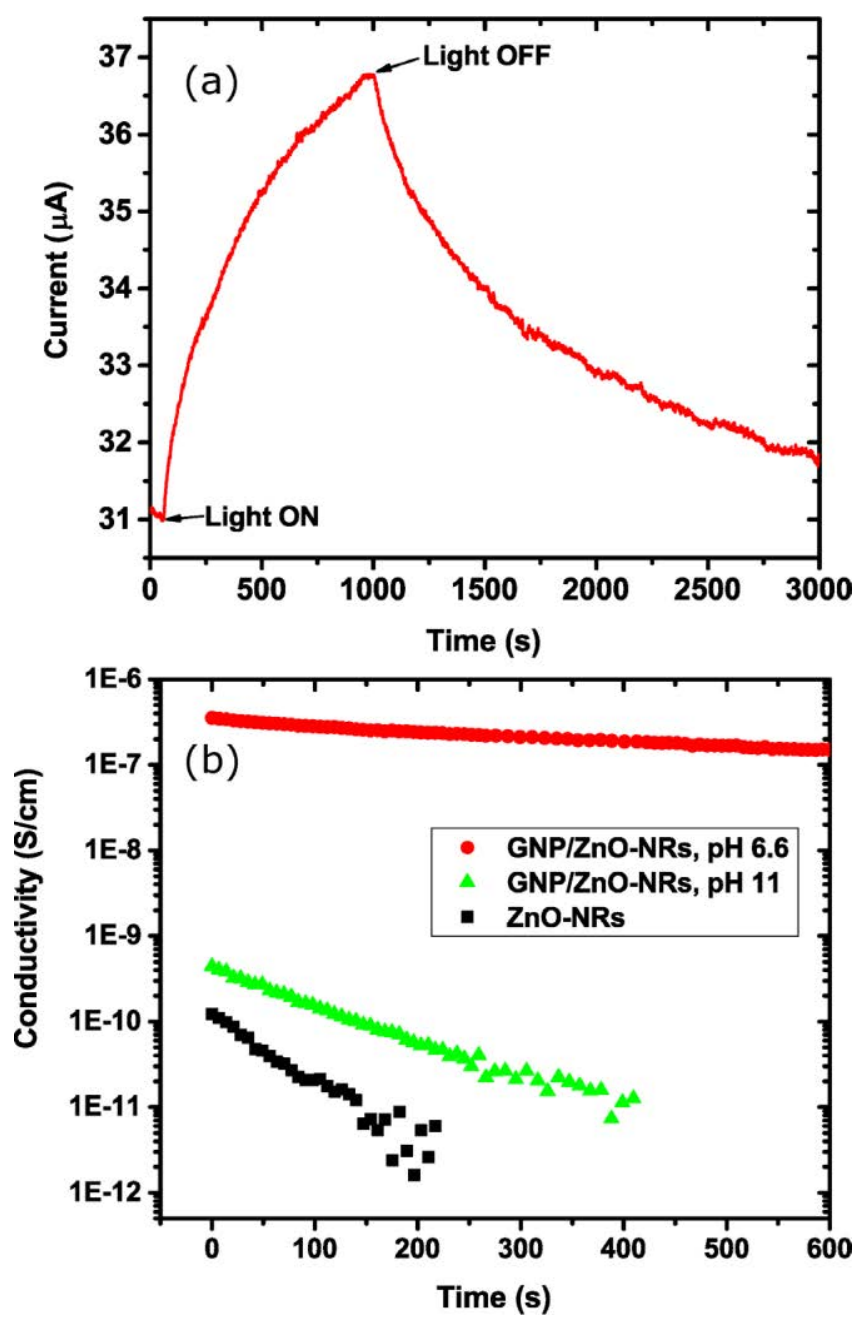

Fig. 11. (a) A typical rise and decay curve for the PPC in GNP/ZnO-NR composites grown at pH 6.6 and (b) time-dependence of the PPC $\left(\sigma_{\mathrm{ph}}\right)$ after switching off the light for three different GNP/ZnO-NRs morphologies, under/after $350 \mathrm{~nm}$ illumination at $300 \mathrm{~K}$ and $1 \mathrm{~V}$ applied bias.

While the ZnO-NRs sample exhibits a long electron lifetime of about 56 $\mathrm{s}$, the composite samples of graphene and $\mathrm{ZnO}$-NRs have much longer electron lifetimes (107 and $877 \mathrm{~s}$ ). The prolonged electron lifetime in these composites can be explained by an efficient transfer of photogenerated electrons to the graphene. This electron injection into the GR causes a strongly reduced recombination rate and thus an enhanced PPC. The sample grown at $\mathrm{pH} 6.6$ shows a significantly longer electron lifetime compared to the sample grown at $\mathrm{pH} 11$. We conclude that the charge transfer between the $\mathrm{ZnO}-\mathrm{NRs}$ and the graphene is more effec- 
tive in the samples with thicker NRs grown at lower $\mathrm{pH}$ compared to the thinner needle-like NRs grown at higher $\mathrm{pH}$. In the next chapter we study the photocatalytic behavior of $\mathrm{GNP} / \mathrm{ZnO}$, based on the charge separation mechanism explained here.

Table 2. Dark conductivity $\left(\sigma_{\mathrm{d}}\right)$, PPC directly after switching off the light $\left(\sigma_{\mathrm{o}}\right)$ and excess electron lifetime $\left(\tau_{d}\right)$ of three different undoped samples at $300 \mathrm{~K}$.

\begin{tabular}{lccc}
\hline & $\sigma_{\mathrm{d}}(\mathrm{S} / \mathrm{cm})$ & $\sigma_{\mathrm{o}}(\mathrm{S} / \mathrm{cm})$ & $\tau_{\mathrm{d}}(\mathrm{s})$ \\
\hline ZnO-NRs & $1.7 \times 10^{-9}$ & $1.1 \times 10^{-10}$ & 56 \\
GNP/ZnO-NRs, pH 11 & $7.3 \times 10^{-10}$ & $3.9 \times 10^{-10}$ & 107 \\
GNP/ZnO-NRs, pH 6.6 & $2.1 \times 10^{-6}$ & $3 \times 10^{-7}$ & 877 \\
\hline
\end{tabular}




\section{Photocatalytic applications}

The GNP/ZnO-NRs nanocomposites, explained in the previous chapter, showed a very promising charge separation capability. This brought up the idea that the nanocomposites can also be used as efficient photocatalysts. However, as we previously saw in the photoconductivity response, the GNP/ZnO-NRs nanocomposites are not active in the visible range. The large bandgap of $\mathrm{ZnO}$ can only absorb incident wavelengths in the UV range. Since photocatalysts are supposed to be driven by solar radiation, they should have a spectral efficiency that matches the broad intensity peak in the visible range emitted by the sun.

To sensitize our nanocomposites to visible light, lower bandgap AgI-NPs were introduced into the nanocomposites. Moreover, to optimize the photocatalytic performance the $\mathrm{ZnO}$ structure was changed from NRs to NPs and the weight ratio of GNPs to $\mathrm{ZnO}$ decreased to only $1 \%$.

This chapter contains a summary of the results from in-depth characterization of $\mathrm{ZnO} / \mathrm{GNP} / \mathrm{Ag} / \mathrm{AgI}$ nanocomposites by a variety of experimental techniques. In particular, the photocatalytic properties under simulated solar light were investigated at length. Three different weight ratios of $\mathrm{ZnO} / \mathrm{GNP}$ to $\mathrm{Ag} / \mathrm{AgI}$ of $\mathrm{X}=10 \%, 20 \%$ and $30 \%$ were evaluated for the highest photodegradation efficiency.

\subsection{Characterization of $\mathrm{ZnO} / \mathrm{GNP} / \mathrm{Ag} / \mathrm{AgI}$ nanocomposites}

The crystalline quality of the $\mathrm{ZnO} / \mathrm{GNP} / \mathrm{Ag} / \mathrm{AgI}$ samples was studied using powder x-ray diffraction (XRD) analysis. The XRD data can be found in the Appendix of Paper II, Figure 2. The XRD data showed clear signals from the (001), (002) and (101) crystal planes, indicating a good quality of the pure hexagonal wurtzite ZnO NPs. The XRD data showed no peaks related to GNPs. Clear signals were observed from the cubic crystal planes of AgI. Also, a distinct XRD peak at $2 \theta=77^{\circ}$ indicates the presence of reduced silver atoms $\left(\mathrm{Ag}^{\circ}\right)$ in the samples. The mechanism of forming these Ag-NPs was previously explained in Chapter 2. 
The field-emission scanning electron microscopy (FE-SEM) image in Fig. 12 and transmission electron microscopy (TEM) image in Fig. 13, confirm a uniform conjunction of ZnO-NPs and GNPs in the $\mathrm{ZnO} / \mathrm{GNP} / \mathrm{Ag} / \mathrm{AgI}$ nanocomposite. The GNPs act as a substructure to assemble the NPs and also serve as an electrical bridge between them. This can have a positive effect on the charge separation at the $\mathrm{GNP} / \mathrm{ZnO}$-NPs interface that will be discussed below.

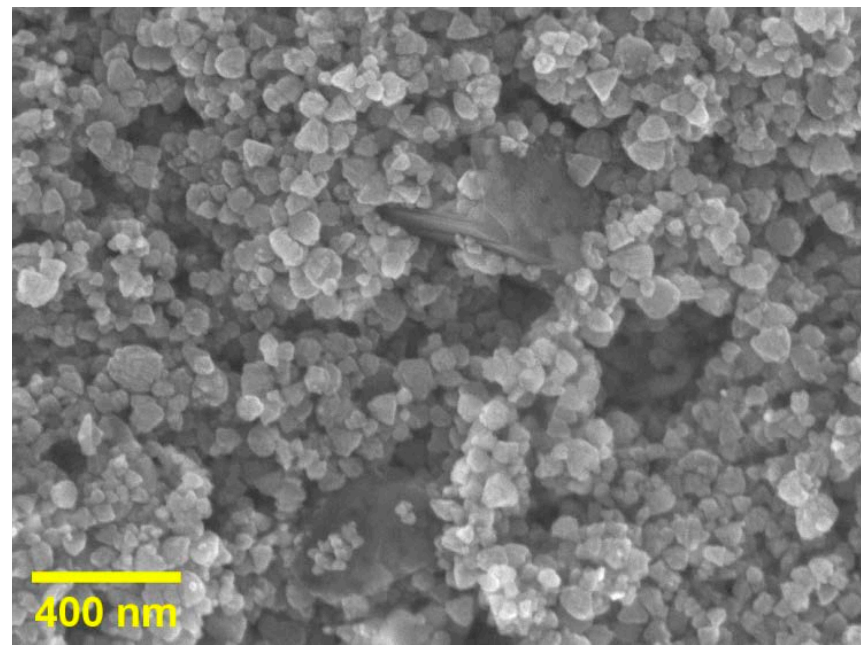

Fig. 12. FE-SEM image of a $\mathrm{ZnO} / \mathrm{GNP} / \mathrm{Ag} / \mathrm{AgI}(20 \%)$ nanocomposite.

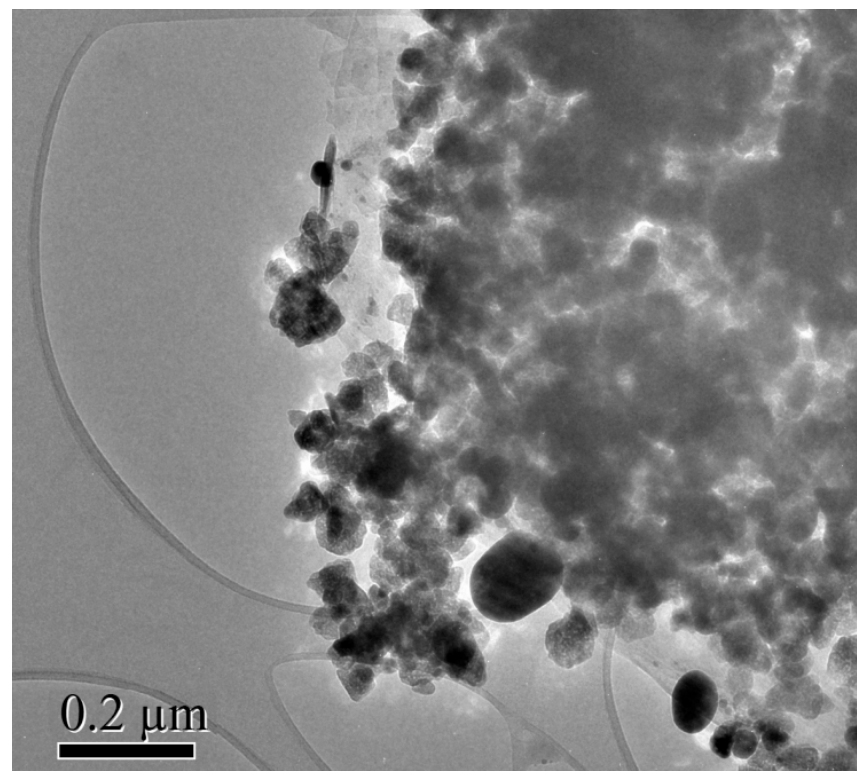

Fig. 13. TEM image of a $\mathrm{ZnO} / \mathrm{GNP} / \mathrm{Ag} / \mathrm{AgI}(20 \%)$ nanocomposite. 
The elemental distribution of the $\mathrm{ZnO} / \mathrm{GNP} / \mathrm{Ag} / \mathrm{AgI}$ (20\%) nanocomposites were examined using scanning TEM (STEM) high-angle annular dark-field (HAADF) imaging (Fig. 14(a)) and STEM-EDS elemental mapping (Fig. 14(b-d)). The presence of $\mathrm{Zn}, \mathrm{O}$ and localized $\mathrm{Ag} / \mathrm{I}$ was confirmed. The elemental distribution in Fig. 14(c) shows two displaced phases of $\mathrm{Ag}$ and I, indicating reduced $\mathrm{Ag}^{\circ}$ atoms on the AgI-NPs. The mechanism of forming these Ag-NPs was previously explained in Chapter 2. The well distributed elements in the nanocomposite cause a stronger bonding and leads to higher photodegradation efficiency.

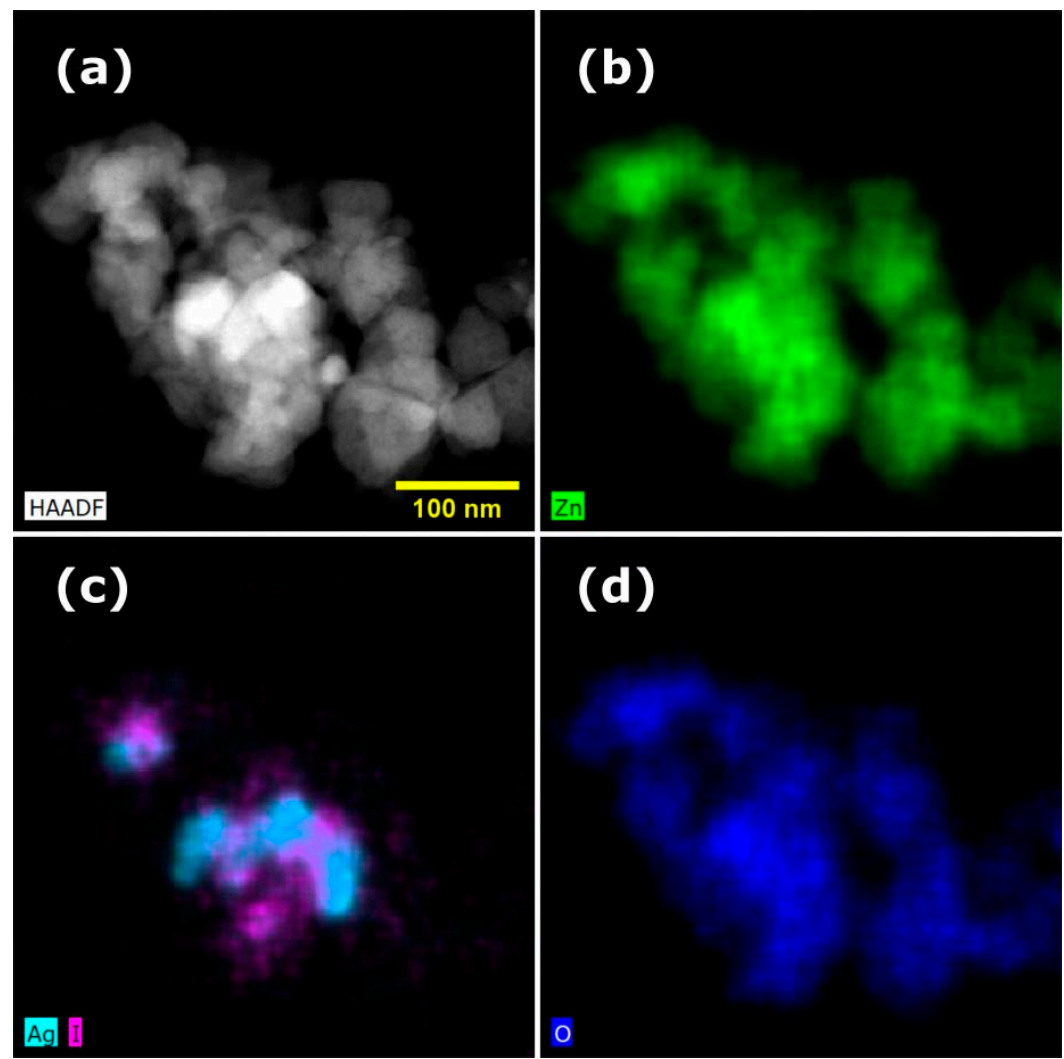

Fig. 14. (a) STEM-HAADF image of a $\mathrm{ZnO} / \mathrm{GNP} / \mathrm{Ag} / \mathrm{AgI}$ (20\%) nanocomposite. (b-d) Corresponding STEM-EDS elemental maps displaying $\mathrm{Zn}, \mathrm{Ag} / \mathrm{I}$ and $\mathrm{O}$ distributions, respectively.

\subsection{Elemental bonding and chemical shifts in XPS}

To precisely investigate the elemental bonding in the nanocomposites, $\mathrm{x}$-ray photoelectron spectroscopy (XPS) analysis was employed. Here in this section, a background to XPS analysis, binding energy and its rela- 
tion to the chemical shifts in XPS data will be discussed. More experimental details about the analyzed samples can be found in the Appendix of Paper II.

\subsubsection{XPS background}

The history of XPS dates back to the photoelectric effect, discovered in 1887 by Heinrich Hertz and investigated further by his assistant Philipp Lenard in 1900-1902. In the photoelectric effect, monochromatic light impinging on a sample causes electrons (so called photoelectrons) to be emitted if the photon energy is higher than the work function $\left(\varphi_{0}\right)$ of the sample. The kinetic energy (EK) of the emitted photoelectrons depends on the binding energy $\left(\mathrm{E}_{\mathrm{B}}\right)$ of the electrons in the material and the photon energy (hv) of the incident light according to:

$$
E_{K}=\mathrm{h} v-E_{B}
$$

$\mathrm{E}_{B}$ is the energy difference between the total ground state energy of an atom, and the total energy of the cation with a core hole. In XPS, x-ray radiation with known photon energy (hv) is shone on the sample under ultra-high vacuum (UHV) conditions (Fig. 15). According to the photoelectric effect, electrons with different EK are emitted from the sample and detected by a spectrometer. Subsequently, the binding energy $\left(\mathrm{E}_{\mathrm{B}}\right)$ of these electrons is calculated based on the Einstein equation (Eq. (1)).

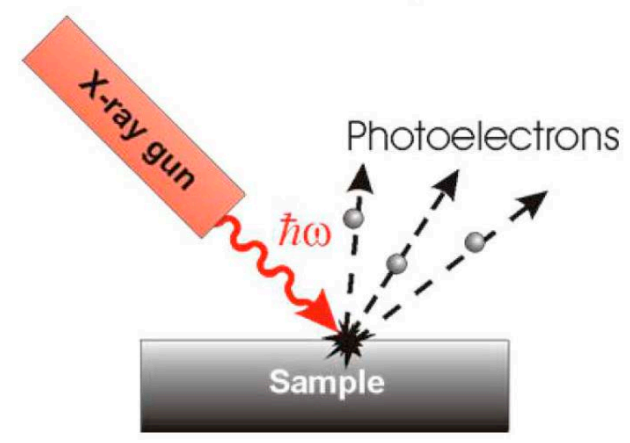

Fig. 15. Schematic of XPS

In XPS, only the core level electrons (the electrons that are tightly bound to the nucleus and not participating in the chemical bonding) with binding energy smaller than the energy of the x-ray source can be detected. $\mathrm{E}_{\mathrm{B}}$ is usually between $\mathrm{o}$ to $1200 \mathrm{eV}$ for a typical (Al Ka) x-ray source.

Each element has as unique set of binding energies, and thus peaks in the XPS spectra, that work as a finger print for that element. By comparing these peaks to a database with tabulated elements, the pres- 
ence of each element can be identified in the sample. Fig. 16 shows an XPS survey scan spectrum for a $\mathrm{ZnO} / \mathrm{GNP} / \mathrm{Ag} / \mathrm{AgI}$ (20\%) nanocomposite with clear peaks for $\mathrm{C}, \mathrm{Ag}, \mathrm{O}$, I and $\mathrm{Zn}$ atoms.

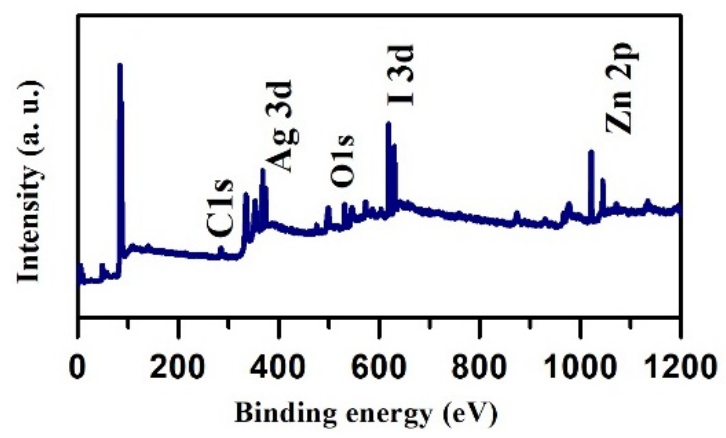

Fig. 16. An XPS survey scan spectrum for a $\mathrm{ZnO} / \mathrm{GNP} / \mathrm{Ag} / \mathrm{AgI}$ (20\%) nanocomposite.

The core levels are denoted as below in Fig. 17, where $\mathrm{n}$ is the principal quantum number describing the basic energy levels of an atom, $l$ is the angular momentum quantum number, $s$ is the spin quantum number and $\mathrm{j}$ is the total angular momentum number $\left(\mathrm{j}=\mathrm{l}_{ \pm \mathrm{s}}\right)$.

Although the penetration depth of x-ray radiation in most materials is comparably large (in $\mu \mathrm{m}$ range), XPS analysis is extremely surface sensitive. The limiting factor is the average distance for photoelectrons to leave the sample without losing their energy. This average distance from the surface is usually less than $1 \mathrm{~nm}$. Thus, XPS data only conveys information about some multiple surface layers of the sample. The lateral resolution is relatively low and in the order of $150 \mathrm{~nm}$ to $15 \mu \mathrm{m}$. XPS has a very good detection limit of about 0.1 at\%, and can detect basically all elements except hydrogen.

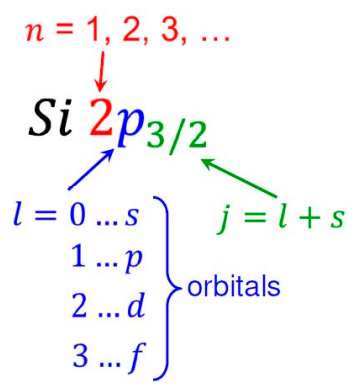

Fig. 17. The core level notation for $\mathrm{Si}$. 


\subsubsection{Core-level binding energy and chemical shift}

The binding energy peaks of an element depend on the local chemical environment around that element, can be a little shifted. Although the core-level electrons are not directly involved in the chemical bonding, the binding energies of the core electrons are influenced by the total charge density in the chemical bonds and electronegativity of neighboring atoms. Typically, a lower charge density around an atom results in higher binding energies of the core-levels.

As an example, the chemical shifts of the core-level binding energy of the four different $\mathrm{C}$ atoms in the trifluoro-acetate molecule are shown in Fig. 18. The first $\mathrm{C}$ atom from the right side is bonded to similar electronegative atoms (three $\mathrm{H}$ and a $\mathrm{C}$ atom) without any binding energy shift. The second $\mathrm{C}$ atom is bonded to a more electronegative $\mathrm{O}$ atom, and consequently has a lower charge density, causing higher binding energy. The third and the fourth $\mathrm{C}$ atoms are also bonded to more electronegative atoms, making it harder to pull out further electrons i.e. the binding energies increase. High-resolution XPS measurements can determine accurate chemical shifts in binding energies, which makes this kind of spectroscopy extremely powerful for chemical analysis of complex samples e.g. nanocomposites.

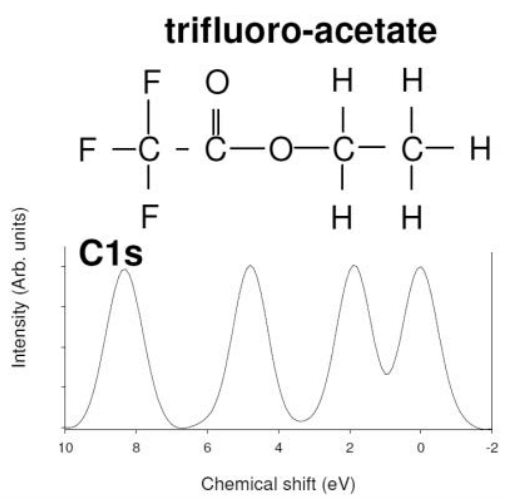

Fig. 18. The chemical shifts of the core-level binding energy for $\mathrm{C}$ 1s atoms in the trifluoro-acetate molecule [49].

\subsubsection{XPS analysis of the ZnO/GNP/Ag/AgI samples}

The presence of the main elements $\mathrm{C}, \mathrm{Ag}, \mathrm{O}, \mathrm{I}$ and $\mathrm{Zn}$ was identified by the XPS survey scan spectrum for the $\mathrm{ZnO} / \mathrm{GNP} / \mathrm{Ag} / \mathrm{AgI}$ (20\%) sample, as shown in Fig. 16. High resolution XPS scans for each of these elements reveals more information about the chemical bonding between them. The illustrated XPS data in the Appendix of Paper II 2, shows three types of chemical bonding for the $\mathrm{C}$ atom, corresponding to $\mathrm{C}-\mathrm{C}$, 
$\mathrm{C}-\mathrm{O}-\mathrm{C}$ and $\mathrm{C}=\mathrm{O}$. Two different $\mathrm{O}$ bonds to $\mathrm{Zn}$ atoms and to hydroxyl groups were also detected. The sample also included two species of Ag atoms, the $\mathrm{Ag}^{+}$at lower binding energy and the reduced $\mathrm{Ag}^{\circ}$ at higher binding energy.

\subsection{Photocatalytic performance of the $\mathrm{ZnO} / \mathrm{GNP} / \mathrm{Ag} / \mathrm{AgI}$ nanocomposites}

The photocatalytic performance of the synthesized samples was studied by degradation of Congo red (CR) dye as an organic molecule, under simulated solar irradiation. The degradation of CR dye was measured by time-resolved UV-Vis absorbance spectroscopy on a mixture of the nanocomposite as photocatalyst and the CR dye solution.

The optical characterization of the $\mathrm{ZnO} / \mathrm{GNP} / \mathrm{Ag} / \mathrm{AgI}$ nanocomposite was performed using UV-Vis absorbance spectroscopy and cathodoluminescence (CL). The UV-Vis absorbance spectra showed absorption peaks at about $380 \mathrm{~nm}$ and $430 \mathrm{~nm}$ attributed to bandgap excitation in $\mathrm{ZnO}$ and to surface plasmon absorption induced by the $\mathrm{Ag} / \mathrm{AgI}$ NPs, respectively. The Ag/AgI surface plasmon-enhanced luminescence at about $430 \mathrm{~nm}$ was confirmed by CL spectroscopy. More details are found in the Appendix of Paper II.

\subsubsection{Photodegradation efficiency of CR dye}

The photocatalytic activities of $\mathrm{ZnO}, \mathrm{ZnO} / \mathrm{GNP}, \mathrm{ZnO} / \mathrm{Ag} / \mathrm{AgI}$ and $\mathrm{ZnO} / \mathrm{GNP} / \mathrm{Ag} / \mathrm{AgI}$ samples were investigated through the degradation of CR dye under simulated solar irradiation. For these measurements, about $0.05 \mathrm{~g}$ of each sample was mixed with $100 \mathrm{ml}$ of $\mathrm{CR}$ dye solution with the initial concentration of $0.2 \mathrm{~g} / \mathrm{l}$. These mixtures were stirred for 30 minutes in dark to reach the adsorption-desorption equilibrium between the photocatalyst and the dye molecules. Subsequently, the mixture was irradiated for $60 \mathrm{~min}$ in $15 \mathrm{~min}$ interval steps. At each step, the absorption spectrum of the remaining CR dye in the solution was recorded.

The CR dye has an absorption peak at $497 \mathrm{~nm}$ which decreases in height with irradiation time, verifying the degradation of the CR dye. In fact, according to the Beer-Lambert law, the absorption is proportional to the dye concentration in the solution. This allows us to define the photodegradation efficiency, using absorbance instead of the concentrations according to Eq. (2).

$$
\text { Degradation }(\%)=\frac{A_{0}-A}{A_{0}} \times 100
$$


Here, $A_{o}$ is the initial absorbance of the dye and $A$ is the absorbance after a specific irradiation time. In the Appendix of Paper II, the photodegradation efficiency of the $\mathrm{ZnO}, \mathrm{ZnO} / \mathrm{GNP}, \mathrm{ZnO} / \mathrm{Ag} / \mathrm{AgI}$ and $\mathrm{ZnO} / \mathrm{GNP} / \mathrm{Ag} / \mathrm{AgI}$ samples have been compared. Briefly summarized; i) in the absence of any photocatalyst, the degradation of the CR is negligible, ii) the plasmonic $\mathrm{ZnO} / \mathrm{GNP} / \mathrm{Ag} / \mathrm{AgI}$ nanocomposites show the highest photocatalytic activity among all samples and iii) the plasmonic nanocomposite with $20 \% \mathrm{Ag} / \mathrm{AgI}$ weight ratio has the highest photodegradation activity (Fig. 19).

The degradation rate constant can be calculated based on the Langmuir-Hinshelwood's pseudo-first order kinetics model, according to Eq. (3), where $t$ is the irradiation time $(\mathrm{min}), \mathrm{C}_{o}$ is the initial concentration (mol. $\left.\mathrm{l}^{-1}\right)$ of the dye, and $\mathrm{C}(\mathrm{t})$ is the concentration $\left(\mathrm{mol.} \mathrm{l}^{-1}\right)$ of the dye after irradiation time $t$.

$$
\text { Degradation rate constant }=\frac{\ln \left[\frac{C_{0}}{C(t)}\right]}{\mathrm{t}}
$$

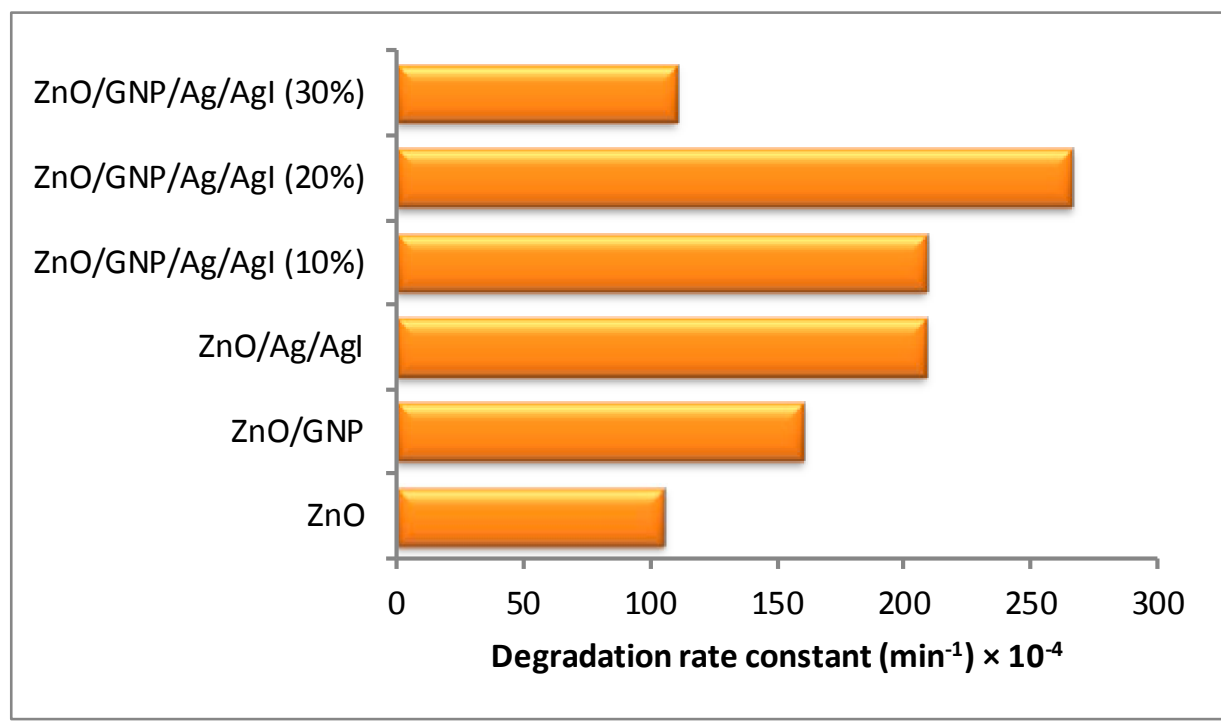

Fig. 19. The degradation rate constant of CR dye for different samples.

\subsubsection{Photocatalytic mechanism}

A schematic of the proposed photocatalytic mechanism is shown in the Appendix of Paper II. In the presence of solar irradiation, both $\mathrm{ZnO}$ and AgI can absorb light at different wavelengths and generate electrons and holes in their respective conduction bands (CB) and valence bands (VB). Due to the band offsets, the electrons residing in the CB of AgI 
migrate to the $\mathrm{CB}$ of $\mathrm{ZnO}$ and, in a likewise manner, holes in the $\mathrm{VB}$ of $\mathrm{ZnO}$ move to the VB of AgI, which promotes an efficient charge separation of the photogenerated $\mathrm{e} / \mathrm{h}$ pairs which prevents recombination. Subsequently, the photogenerated electrons are transferred from the $\mathrm{CB}$ of the ZnO-NPs to the GNPs acting as an electron sink. The transport of electrons to the GNPs leads to a further reduction of the probability of $\mathrm{e} / \mathrm{h}$ recombination. This strongly enhances the photocatalytic efficiency. In addition, the plasmonic Ag-NPs act as efficient generators of electrons and holes in the visible range via the LSPR effect.

The separated electrons react with oxygen molecules to produce superoxide radicals that subsequently get converted into hydroxyl radicals through multi-electron reduction reactions. Finally, these active species can react with pollutant (dye) molecules and decompose them into $\mathrm{H}_{2} \mathrm{O}, \mathrm{CO}_{2}$, and mineral compounds.

The photogenerated holes can directly oxidize the adsorbed dye molecules to produce degradation products. Summarized, the high photodegradation efficiency of $\mathrm{ZnO} / \mathrm{GNP} / \mathrm{Ag} / \mathrm{AgI}$ nanocomposites is attributed to the uniform distribution of NPs at the surface of the GNPs, and to strong synergy effects between GNPs, ZnO-NPs and Ag/AgI NPs that favors interfacial charge transfer that prevents $\mathrm{e} / \mathrm{h}$ recombination. 


\section{Conclusions}

This thesis deals with hydrothermal solution-based synthesis and characterization of GNP/ZnO nanocomposites. Growth and in-situ doping of conjugated GNP/ZnO-NRs nanocomposites with two different morphologies of $\mathrm{ZnO}-\mathrm{NRs}$ were realized, and compared with respect to dispersity, GNP/ZnO-NRs bonding quality and conductivity. The samples grown at higher $\mathrm{pH}$ showed better dispersion and improved bonding quality between the GNPs and NRs compared to samples grown at lower $\mathrm{pH}$, but lower electrical conductivity. Furthermore, an improved charge separation was observed in the GNP/ZnO composites, compared to bare $\mathrm{ZnO}$-NRs. The $\mathrm{ZnO}-\mathrm{NRs}$, grown on the GNPs, act as spacers that introduce porosities which significantly promotes the capability to form more complex composites with other materials.

$\mathrm{ZnO} / \mathrm{GNP} / \mathrm{Ag} / \mathrm{AgI}$ nanocomposites were prepared to verify their excellent photocatalytic performance under simulated solar irradiation. The addition of $\mathrm{Ag} / \mathrm{AgI}$ to the structure leads to significantly increased photocatalytic performance, compared to pristine $\mathrm{ZnO}$ and $\mathrm{ZnO} / \mathrm{GNP}$ samples. This enhancement was attributed to increased light absorption in the visible range and an effective charge separation of the photogenerated carriers, suppressing e/h recombination. A maximum photodegradation efficiency of about $90 \%$ was measured after $60 \mathrm{~min}$. solar irradiation of the CR dye. Summarized, the results show a promising potential of our developed nanocomposites for applications in printable electronics and photoelectrochemistry. 


\title{
Outlook
}

\begin{abstract}
All the discussed nanostructures in this thesis were synthesized by a random and substrate-free growth procedure. This method is very attractive from the perspectives of simplicity and cost effectiveness, but suffers from the drawback that a precise controlling of the nanostructures is extremely difficult. The $\mathrm{ZnO}-\mathrm{NRs}$ grow randomly in any direction with a large distribution of sizes. This makes it very hard to fabricate e.g. functional devices comprising large arrays of NRs with welldefined contacts.

For the next step, vertically well-aligned $\mathrm{ZnO}$-NRs should be grown on a patterned substrate. The diameter and the length of the grown ZnO-NRs should be accurately controlled. A systematic study is required for preparation of such substrates, in particular the formation of a proper patterned seeding layer. Nanoimprint lithography (NIL) is an option to create the desired patterned substrates. Electrodeposition (ED) can be used to selectively seed the openings in the pattern. The ED parameters and the $\mathrm{ZnO}$-NRs growth parameters need to be optimized. Subsequently, the prepared well-aligned $\mathrm{ZnO}$-NRs structures can be integrated with graphene for more advanced optoelectronic device fabrication such as, solar cells, photodetectors, RF components, and biosensors.
\end{abstract}




\section{References}

[1] Ertekin E, Greaney P A, Sands T D and Chrzan D C 2002 Equilibrium Analysis of Lattice-Mismatched Nanowire Heterostructures MRS Online Proceedings Library Archive 737

[2] Gao N and Fang X 2015 Synthesis and Development of Graphene-Inorganic Semiconductor Nanocomposites Chem. Rev. 115 8294-343

[3] Di Bartolomeo A 2016 Graphene Schottky diodes: An experimental review of the rectifying graphene/semiconductor heterojunction Physics Reports 606 1-58

[4] Jariwala D, Marks T J and Hersam M C 2017 Mixed-dimensional van der Waals heterostructures Nature Materials 16 170-81

[5] Weiss N O, Zhou H, Liao L, Liu Y, Jiang S, Huang Y and Duan X 2012 Graphene: An Emerging Electronic Material Adv. Mater. 24 5782-825

[6] Anon 2017 Nanotechnologies-Vocabulary-Part 13: Graphene and Related Two-Dimensional (2D) Materials

[7] Anon 2017 Characterisation of the structure of graphene

[8] Gee C-M, Tseng C-C, Wu F-Y, Chang H-P, Li L-J, Hsieh Y-P, Lin $\mathrm{C}-\mathrm{T}$ and Chen J-C 2013 Flexible transparent electrodes made of electrochemically exfoliated graphene sheets from low-cost graphite pieces Displays 34 315-9

[9] Arapov K, Rubingh E, Abbel R, Laven J, de With G and Friedrich H 2016 Conductive Screen Printing Inks by Gelation of Graphene Dispersions Adv. Funct. Mater. 26 586-93

[10] Klingshirn C, Fallert J, Zhou H, Sartor J, Thiele C, Maier-Flaig F, Schneider D and Kalt H 201065 years of $\mathrm{ZnO}$ research - old and very recent results phys. stat. sol. (b) 247 1424-47

[11] Özgür Ü, Alivov Y I, Liu C, Teke A, Reshchikov M A, Doğan S, Avrutin V, Cho S-J and Morkoç H 2005 A comprehensive review of $\mathrm{ZnO}$ materials and devices Journal of Applied Physics 98 041301 
[12] Hsu C-H and Chen D-H 2010 Synthesis and conductivity enhancement of Al-doped $\mathrm{ZnO}$ nanorod array thin films Nanotechnology 21285603

[13] Hagendorfer H, Lienau K, Nishiwaki S, Fella C M, Kranz L, Uhl A R, Jaeger D, Luo L, Gretener C, Buecheler S, Romanyuk Y E and Tiwari A N 2014 Highly Transparent and Conductive ZnO: Al Thin Films from a Low Temperature Aqueous Solution Approach Adv. Mater. 26 632-6

[14] Jia J, Takasaki A, Oka N and Shigesato Y 2012 Experimental observation on the Fermi level shift in polycrystalline Al-doped $\mathrm{ZnO}$ films Journal of Applied Physics 112013718

[15] Bamiduro O, Mustafa H, Mundle R, Konda R B and Pradhan A K 2007 Metal-like conductivity in transparent Al:ZnO films Appl. Phys. Lett. 90252108

[16] Nian Q, Callahan M, Look D, Efstathiadis H, Bailey J and Cheng G J 2015 Highly transparent conductive electrode with ultra-low HAZE by grain boundary modification of aqueous solution fabricated alumina-doped zinc oxide nanocrystals APL Materials 3 062803

[17] Li D, Muller M B, Gilje S, Kaner R B and Wallace G G 2008 Processable aqueous dispersions of graphene nanosheets Nat Nano 3 101-5

[18] Kim Y-J, Lee J-H and Yi G-C 2009 Vertically aligned ZnO nanostructures grown on graphene layers Applied Physics Letters 95213101

[19] Choi W M, Shin K-S, Lee H S, Choi D, Kim K, Shin H-J, Yoon SM, Choi J-Y and Kim S-W 2011 Selective growth of $\mathrm{ZnO}$ nanorods on $\mathrm{SiO} 2 / \mathrm{Si}$ substrates using a graphene buffer layer Nano Res. 4 440-7

[20] Kim Y-J, Hadiyawarman, Yoon A, Kim M, Yi G-C and Liu C 2011 Hydrothermally grown $\mathrm{ZnO}$ nanostructures on few-layer graphene sheets Nanotechnology 22245603

[21] Chandraiahgari C R, Bellis G D, Balijepalli S K, Kaciulis S, Ballirano P, Migliori A, Morandi V, Caneve L, Sarto F and Sarto M S 2016 Control of the size and density of $\mathrm{ZnO}$-nanorods grown onto graphene nanoplatelets in aqueous suspensions RSC Adv. 6 83217-25

[22] Munshi A M, Dheeraj D L, Fauske V T, Kim D-C, van Helvoort A T J, Fimland B-O and Weman H 2012 Vertically Aligned GaAs Nanowires on Graphite and Few-Layer Graphene: Generic Model and Epitaxial Growth Nano Lett. 12 4570-6 
[23] Larson K, Clark A, Appel A, Dai Q, He H and Zygmunt S 2015 Surface-dependence of interfacial binding strength between zinc oxide and graphene RSC Advances 5 65719-24

[24] Lin J, Penchev M, Wang G, Paul R K, Zhong J, Jing X, Ozkan M and Ozkan C S 2010 Heterogeneous Graphene Nanostructures: $\mathrm{ZnO}$ Nanostructures Grown on Large-Area Graphene Layers Small 6 2448-52

[25] Hwang J O, Lee D H, Kim J Y, Han T H, Kim B H, Park M, No K and Kim S O 2011 Vertical $\mathrm{ZnO}$ nanowires/graphene hybrids for transparent and flexible field emission J. Mater. Chem. $213432-7$

[26] Li G, Wang Y and Mao L 2014 Recent progress in highly efficient Ag-based visible-light photocatalysts $R S C$ Adv. 4 53649-61

[27] Pirhashemi M and Habibi-Yangjeh A 2017 Preparation of novel nanocomposites by deposition of $\mathrm{Ag}_{2} \mathrm{WO}_{4}$ and $\mathrm{AgI}$ over $\mathrm{ZnO}$ particles: Efficient plasmonic visible-light-driven photocatalysts through a cascade mechanism Ceramics International 43 1344760

[28] Reddy D A, Lee S, Choi J, Park S, Ma R, Yang H and Kim T K 2015 Green synthesis of AgI-reduced graphene oxide nanocomposites: Toward enhanced visible-light photocatalytic activity for organic dye removal Applied Surface Science 341 175-84

[29] Fuchs P, Hagendorfer H, Romanyuk Y E and Tiwari A N 2015 Doping strategies for highly conductive $\mathrm{Al}$-doped $\mathrm{ZnO}$ films grown from aqueous solution Phys. Status Solidi A 212 51-5

[30] Verrier C, Appert E, Chaix-Pluchery O, Rapenne L, Rafhay Q, Kaminski-Cachopo A and Consonni V 2017 Tunable Morphology and Doping of $\mathrm{ZnO}$ Nanowires by Chemical Bath Deposition Using Aluminum Nitrate J. Phys. Chem. C 121 3573-83

[31] Verrier C, Appert E, Chaix-Pluchery O, Rapenne L, Rafhay Q, Kaminski-Cachopo A and Consonni V 2017 Effects of the pH on the Formation and Doping Mechanisms of $\mathrm{ZnO}$ Nanowires Using Aluminum Nitrate and Ammonia Inorg. Chem. 56 13111-22

[32] Miyake M, Fukui H and Hirato T 2012 Preparation of Al-doped $\mathrm{ZnO}$ films by aqueous solution process using a continuous circulation reactor Phys. Status Solidi A 209 945-8

[33] Edinger S, Bansal N, Bauch M, Wibowo R A, Hamid R, Trimmel G and Dimopoulos T 2017 Comparison of chemical bath-deposited ZnO films doped with Al, Ga and In J Mater Sci 52 9410-23

[34] Smits F M 1958 Measurement of Sheet Resistivities with the Four-Point Probe Bell System Technical Journal 37 711-8 
[35] Gfroerer T H 2006 Photoluminescence in Analysis of Surfaces and Interfaces Encyclopedia of Analytical Chemistry (John Wiley \& Sons, Ltd)

[36] Alnoor H, Pozina G, Khranovskyy V, Liu X, Iandolo D, Willander $\mathrm{M}$ and Nur O 2016 Influence of $\mathrm{ZnO}$ seed layer precursor molar ratio on the density of interface defects in low temperature aqueous chemically synthesized $\mathrm{ZnO}$ nanorods/GaN light-emitting diodes Journal of Applied Physics 119165702

[37] Sakurai M, Wang Y G, Uemura T and Aono M 2009 Electrical properties of individual $\mathrm{ZnO}$ nanowires Nanotechnology 20 155203

[38] Zhu Q, Xie C, Li H, Yang C, Zhang S and Zeng D 2014 Selectively enhanced UV and NIR photoluminescence from a degenerate $\mathrm{ZnO}$ nanorod array film Journal of Materials Chemistry C 2 4566-80

[39] Abdolahzadeh Ziabari A and Rozati S M 2012 Carrier transport and bandgap shift in n-type degenerate $\mathrm{ZnO}$ thin films: The effect of band edge nonparabolicity Physica B: Condensed Matter $\mathbf{4 0 7}$ 4512-7

[40] Wang M, Lee K E, Hahn S H, Kim E J, Kim S, Chung J S, Shin E $\mathrm{W}$ and Park C 2007 Optical and photoluminescent properties of sol-gel Al-doped $\mathrm{ZnO}$ thin films Materials Letters 61 1118-21

[41] Rahman M M, Khan M K R, Islam M R, Halim M A, Shahjahan M, Hakim M A, Saha D K and Khan J U 2012 Effect of Al Doping on Structural, Electrical, Optical and Photoluminescence Properties of Nano-Structural ZnO Thin Films Journal of Materials Science \& Technology $\mathbf{2 8} 329-35$

[42] Chalangar E, Machhadani H, Lim S-H, Karlsson K F, Nur O, Magnus Willander and Pettersson H 2018 Influence of morphology on electrical and optical properties of graphene/Al-doped $\mathrm{ZnO}-$ nanorod composites Nanotechnology 29415201

[43] Medved D B 1961 Photoconductivity and chemisorption kinetics in sintered zinc oxide semiconductor* Journal of Physics and Chemistry of Solids 20 255-67

[44] Chen M-W, Chen C-Y, Lien D-H, Ding Y and He J-H 2010 Photoconductive enhancement of single $\mathrm{ZnO}$ nanowire through localized Schottky effects Opt. Express, OE 18 14836-41

[45] Moore J C and Thompson C V 2013 A Phenomenological Model for the Photocurrent Transient Relaxation Observed in $\mathrm{ZnO}$-Based Photodetector Devices Sensors (Basel) 13 9921-40 
[46] Zhu Q, Xie C, Li H, Zhang J and Zeng D 2015 Through-Process Analytical Modeling of Photoconductance Spectrum for Porous ZnO Nanocrystalline Film Chem. Mater. 27 2861-74

[47] Zhu Q, Xie C, Li H and Zeng D 2016 A method for modeling and deciphering the persistent photoconductance and long-term charge storage of ZnO nanorod arrays Nano Res. 9 2972-3002

[48] Rani M and Tripathi S K 2016 Electron transfer properties of organic dye sensitized $\mathrm{ZnO}$ and $\mathrm{ZnO} / \mathrm{TiO} 2$ photoanode for dye sensitized solar cells Renewable and Sustainable Energy Reviews 61 97-107

[49] Gelius U, Basilier E, Svensson S, Bergmark T and Siegbahn K 1973 A high resolution ESCA instrument with X-ray monochromator for gases and solids Journal of Electron Spectroscopy and Related Phenomena 2 405-34 
Part II: PAPERS 


\section{Papers}

The papers associated with this thesis have been removed for copyright reasons. For more details about these see:

http://urn.kb.se/resolve?urn=urn:nbn:se:liu:diva-157095 


\section{FACULTY OF SCIENCE AND ENGINEERING}

Linköping Studies in Science and Technology, Licentiate Thesis No. 1847

Department of Science and Technology

Linköping University

SE-581 83 Linköping, Sweden

www.liu.se 